\title{
Admissible Linear Map Models of Linear Cameras
}

\author{
Guillaume Batog* \\ Université de Nancy 2
}

\author{
Xavier Goaoc* \\ INRIA Nancy Grand Est
}

\author{
Jean Ponce $^{\dagger}$ \\ École Normale Supérieure
}

\begin{abstract}
This paper presents a complete analytical characterization of a large class of central and non-central imaging devices dubbed linear cameras by Ponce [10]. Pajdla [8] has shown that a subset of these, the oblique cameras, can be modelled by a certain type of linear map. We give here a full tabulation of all admissible maps that induce cameras in the general sense of Grossberg and Nayar [5], and show that these cameras are exactly the linear ones. Combining these two models with a new notion of intrinsic parameters and normalized coordinates for linear cameras allows us to give simple analytical formulas for direct and inverse projections. We also show that the epipolar geometry of any two linear cameras can be characterized by a fundamental matrix whose size is at most $6 \times 6$ when the cameras are uncalibrated, or by an essential matrix of size at most $4 \times 4$ when their internal parameters are known. Similar results hold for trinocular constraints.
\end{abstract}

\section{Introduction}

As noted by Grossberg and Nayar [5], any imaging system, irrespective of its design, can be decomposed into two elements, its optics, which direct and focus light, and its sensing area, or retina, where incoming light energy is measured. Since light travels along straight lines in homogeneous media, it is geometrically convenient and often physically reasonable to model the behavior of a camera by assuming that one can associate with every point $\boldsymbol{x}$ in the field of view a single representative light ray $\ell$. The optics then map (after an arbitrarily complicated optical path) this primary ray onto a secondary one, $\ell^{\prime}$, that intersects the retina at the image $\boldsymbol{y}^{\prime}$ of the point $\boldsymbol{x}$ (Figure 1). It is also convenient - and natural due to the principle of reversibility in geometric optics- to assume that the retinal point $\boldsymbol{y}^{\prime}$ uniquely determines the ray $\ell^{\prime}$, which in turn uniquely de-

\footnotetext{
*Vegas project-team, LORIA, CNRS / INPL / INRIA / UHP / Nancy 2 UMR 7503, Villers-lès-Nancy, France. \{batog, goaoc \} @ loria. fr

†Willow project-team, LIENS, ENS / INRIA / CNRS UMR 8548, Paris, France. Jean.Poncedens. fr J. Ponce was supported in part by the HFIBMR and TRIANGLES ANR grants.
}

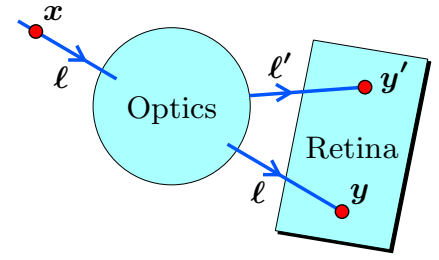

Figure 1: A general model for cameras [5]. For any point $\boldsymbol{x}$, there is a unique primary ray $\ell$ such that light emitted from $\boldsymbol{x}$ along that ray, after beeing refracted and/or reflected along an arbitrarily complicated optical path by the optics, emerges along a secondary ray $\ell^{\prime}$ that strikes the retina at the projection $\boldsymbol{y}^{\prime}$ of $\boldsymbol{x}$. When the internal geometry of the camera is known, one can always assume that the image of the point $\boldsymbol{x}$ is instead located at a point $\boldsymbol{y}$ along the primary ray, with a known mapping from $\boldsymbol{y}$ to $\boldsymbol{y}^{\prime}$.

termines the incoming ray $\ell .{ }^{1}$ As argued in [5], under this "black box" model, the location of a pixel on the retina does not matter (as long as the camera's "internal parameters" are known) and one may choose instead to position the projection of the point $\boldsymbol{x}$ at the point $\boldsymbol{y}$ where the primary ray $\boldsymbol{\ell}$ intersects the retina.

Given this model, let us consider the mapping $\phi$ that associates with any point $\boldsymbol{x}$ in the field of view of a camera the (unique) corresponding ray $\ell$. Again, since light travels along straight lines, this mapping has the property that, for any point $\boldsymbol{y}$ lying on the line $\phi(\boldsymbol{x})$ and in the field of view of the camera, $\phi(\boldsymbol{y})=\phi(\boldsymbol{x})$. Intuitively, this implies that the set of all light rays associated with a camera is in oneto-one continuous correspondence with a two-dimensional surface - that is, it forms a line congruence [11]. ${ }^{2}$ Geometrically, a camera is thus determined by the associated congruence and retina: Given these, the ray $\ell$ associated with a point $\boldsymbol{x}$ is simply the line in the congruence that passes through $\boldsymbol{x}$, and the image $\boldsymbol{y}$ of the point $\boldsymbol{x}$ is the intersection of the line $\ell$ with the retina $r$. Conversely, the preimage $\ell$ of

\footnotetext{
${ }^{1}$ It is of course neither feasible nor desirable to construct an imaging system that collects light along a single light ray at each pixel (after all, one wants to record a finite amount of energy there). This does not take anything away from the idealized model adopted here. For example, the projection geometry of a photographic camera equipped with a lens is effectively modelled, ignoring distortions, by a pinhole camera.

${ }^{2}$ This also follows from the fact that exactly one ray is associated with a retinal point.
} 
any retinal point $\boldsymbol{y}$ is just the line of the congruence passing through it.

Pajdla [8] has shown that the mapping $\phi$ associated with oblique cameras-including bilinear cameras [16], stereo panoramas and cyclographs [12]—can be modelled by a projective map A such that $\phi(\boldsymbol{x})$ is the line joining the points $\boldsymbol{x}$ and $\mathbf{A} \boldsymbol{x}$. On the other hand, Ponce [10] has shown that many central and non-central linear camerasincluding pinhole, two-slit, pushbroom [6], pencil [16] and oblique cameras - can be modelled by linear ${ }^{3}$ line congruences, and used this property to derive general formulas for direct and inverse projections as well as multi-view geometry. This paper unifies the two approaches with the following main contributions:

- We give a simple characterization of the admissible linear maps $A$ such that the corresponding projective maps $\mathbf{A}$ actually define cameras under the model defined in this section (Proposition 4). In turn, this provides a complete tabulation of all admissible maps up to similarity (Table 2).

- We show that Ponce's linear cameras and the cameras that can be defined by an admissible map $A$ are exactly the same (Proposition 5), the linear camera type being determined by the reduced, canonical form of $A$.

- Although, as argued in [10], the role of the retina in the imaging process is secondary for a linear camera, it is more important for cameras associated with rank-4 linear congruences than for pinhole cameras associated with rank-3 line bundles. We make this role explicit, and introduce a notion of intrinsic parameters and normalized coordinates for any linear camera.

- We obtain new and very simple analytic expressions for the direct and inverse projections of any two-slit, pencil or oblique camera, equipped with any generic retinal plane and the corresponding normalized coordinates (Proposition 6).

- We show that the epipolar geometry of any two linear cameras can be characterized by a fundamental matrix of size at most $6 \times 6$ when the cameras are uncalibrated, or by an essential matrix of size at most $4 \times 4$ when their internal parameters are known. A similar result also holds for three-view tensors. Although $6 \times 6$ fundamental matrices have been constructed before for various pairs of linear cameras (see, for example, $[3,10]$ ), this is the first time, to the best of our knowledge, that essential matrices of size at most $4 \times 4$ have been constructed for arbitrary pairs of linear cameras (see [10, 13] for $4 \times 4$ essential matrices involving pairs of two-slit or oblique cameras).

Along the way, we also clarify questions such as the equivalence between retinas for linear cameras (Proposition 1) or the projective equivalence of linear cameras with the same type (Proposition 2), and observe that the type of a linear camera is characterized by what we call its ambiguity lo-

\footnotetext{
${ }^{3}$ The linear dependence of lines is well defined in the framework of classical projective geometry (see $e . g$. [11]).
}

cus - that is, the set of points that belong to several (or none) of the lines in the congruence.

As discussed by Yu et al. [17], linear cameras have potential applications to tasks such as the construction of panoramas, "faux" animations of still scenes, and "cubist" rendering. They also naturally occur as local approximations of general cameras (see [17] for a discussion of linear camera "tessellations").

Paper organization. We start by revisiting in Section 2 the model of linear cameras introduced by Ponce [10]. We then prove in Section 3 the equivalence between linear cameras and those defined by admissible maps after characterizing linear congruences as linear sections of the Plücker quadric. We show in Sections 4 and 5 that combining both representations, linear cameras and admissible maps, yields simple analytical formulas for direct/inverse projections and fundamental matrices. Finally, we conclude in Section 6 with a brief discussion of our results and directions for future research.

Notation. If $x$ is a vector of $\mathbb{R}^{n+1}$ with coordinates $\left(x_{0}, \ldots, x_{n}\right)^{T}$ in the canonical basis of $\mathbb{R}^{n+1}$, we denote by $\boldsymbol{x}$ the corresponding point in $\mathbb{P}^{n}$, identified with its homogeneous coordinates $\left[x_{0}: \ldots: x_{n}\right]$. In general we use bold fonts for projective objects and regular fonts for their linear counterparts, and switch from one to the other representation whenever convenient. We denote by $\vee$ (resp. $\wedge$ ) the "join" (resp. "meet") operator that associates with two points (resp. two planes) the line passing through them (resp. where they intersect). We also often refer to a projective subspace of dimension $k$ as "a $\mathbb{P}^{k}$ " for short.

\section{Linear cameras}

In this section, we revisit the model of linear cameras introduced by Ponce [10]. In particular, we introduce the ambiguity locus, a new geometric attribute of a linear camera, and discuss a projective equivalence of different retinal planes.

As noted previously, the line congruence associated with a camera has the property that exactly one line in the congruence passes through a generic point (multiple lines may pass through certain exceptional points, such as the pinhole of a perspective camera or the slits of a two-slit camera, see Fig. 2). More generally, line congruences can be classified according to their order (the number of lines from the congruence through a generic point) and their class (the number of lines from the congruence contained in a generic plane). Thus the line congruence associated with a camera is what is called a $(1, n)$ congruence - that is, it has order 1 and arbitrary class. Sturm [14] has classified all algebraic $(1, n)$ congruences (see Benić and Gorjanc [1] for an overview of this daunting work). In particular, this classification implies that the linear congruences used by Ponce [10] are exactly the $(1,0)$ and $(1,1)$ congruences, and that at most one line 

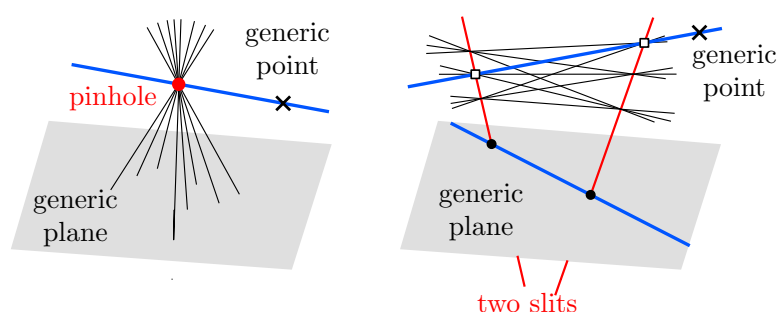

Figure 2: A pinhole camera is defined by a $(1,0)$ congruence (left) and a two-slit camera by a $(1,1)$ congruence (right).

of a linear camera lies in a generic plane (c.f. Fig. 2).

\subsection{Linear congruences and the ambiguity locus}

We define the ambiguity locus of a $(1, n)$ congruence as the set of points that belong to none or more than one line from the congruence. As shown in [11], there are six types of linear congruences, four of which will be of particular interest and can be distinguished by the geometry of their ambiguity locus (Figure 3):

A line bundle is the set of lines through a fixed point. Line bundles are the congruences associated with classical pinhole cameras, and their ambiguity locus is the point common to all lines in the bundle.

An elliptic congruence is the set of real lines intersecting two complex conjugate skew lines. Elliptic congruences are associated with oblique cameras $[8,12,16]$, and their ambiguity locus is empty. The lines in an elliptic congruence can be visualized as a one-parameter family of non-intersecting ruled quadrics (Figure 3, left).

A hyperbolic congruence is the set of lines intersecting two skew lines (Figure 3, middle). Hyperbolic congruences are associated with two-slit and pushbroom cameras $[3,6,18]$, and their ambiguity locus is the pair of lines that intersect all the lines in the congruence.

A parabolic congruence is the set of lines tangent to a non-degenerate ruled quadric along one of its rulings [11, p. 184], forming a one-parameter family of flat pencils sharing one line (Figure 3, right). Parabolic congruences are associated with pencil cameras [16], and their ambiguity locus is the line common to all pencils (or, equivalently, the ruling of the quadric that meets all lines of the congruence).

The remaining congruences are line fields, which consist of all lines in some plane, and degenerate congruences formed by the union of a line bundle and a line field sharing a flat pencil of lines. Line fields do not model interesting cameras since their "field of view" is a plane. It will prove important for technical reasons (e.g., for Proposition 4) to restrict our attention to certain subsets of the lines contained in the remaining linear congruences:

A reduced linear congruence is a two-dimensional set of lines obtained by removing from a linear congruence the

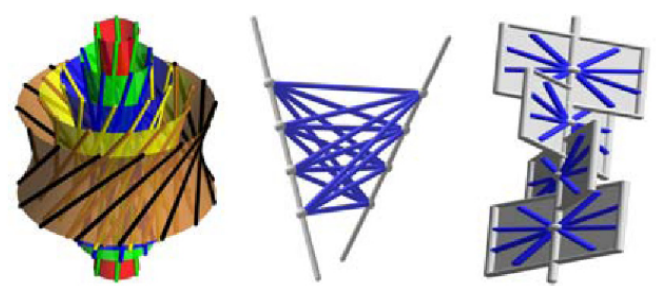

Figure 3: From left to right: elliptic, hyperbolic, and parabolic congruences. Images copyright Hans Havlicek, Vienna University of Technology.

lines that belong (as a set of points) to its ambiguity locus. Thus line bundles, elliptic and hyperbolic congruences are reduced congruences. So is a parabolic congruence minus the line common to all the flat pencils in the corresponding one-parameter family. The reduced congruence associated with a degenerate congruence is a line bundle minus the flat pencil of lines in the associated line field. Finally, line fields are excluded since all their lines belong (as sets of points) to the corresponding ambiguity loci. Reduced congruences are thus line bundles, elliptic, hyperbolic and parabolic congruences from which zero, one, or a one-family parameter of lines have been removed. No line in a reduced linear congruence belongs (as a set of points) to the ambiguity locus of the corresponding congruence. On the other hand, the ambiguity loci associated with a reduced congruence and the original congruence are the same.

Definition: A linear camera is a reduced, non-degenerate, linear congruence together with some retinal plane. This departs from the terminology in [10] where linear cameras are just congruences: Although the retina plays a secondary role in the imaging process (hence the choice of [10]), it will be instrumental in defining intrinsic parameters and normalized coordinates in Section 4.

\subsection{Equivalence of retinal planes}

Call a retina generic for a linear camera with reduced line congruence $\mathcal{L}$ if the number of lines of $\mathcal{L}$ it contains is equal to the class of $\mathcal{L}$. For a pinhole camera, the images formed on any two generic retinal planes are equal up to a projective transformation. This is not true for other types of cameras: Indeed, the points formed by three skew lines of a camera are aligned in any retinal plane containing a transversal to the three lines (there is a two-dimensional set of such planes), but not aligned in any other retinal plane. The corresponding images cannot be projectively equivalent. A weaker form of equivalence does, however, remain.

Proposition 1. Let $\pi_{1}$ and $\pi_{2}$ be the projection functions of two linear cameras with the same underlying reduced linear congruence $\mathcal{L}$ and different (generic) retinal planes $\mathcal{R}_{1}$ and $\mathcal{R}_{2}$ respectively. There exists a projective map $f: \mathcal{R}_{1} \rightarrow$ 
$\mathcal{R}_{2}$ and a projective map $\boldsymbol{g}: \mathbb{P}^{3} \rightarrow \mathbb{P}^{3}$ that preserves $\mathcal{L}$, such that $\boldsymbol{\pi}_{2}=\boldsymbol{f} \circ \boldsymbol{\pi}_{1} \circ \boldsymbol{g}$.

Sketch of proof. Consider two linear cameras with projection functions $\boldsymbol{\pi}_{1}$ and $\boldsymbol{\pi}_{2}$ and underlying linear congruence $\mathcal{L}$. If $\mathcal{L}$ is a line bundle then the statement holds with $\boldsymbol{g}=$ id. Otherwise, $\mathcal{L}$ is a $(1,1)$-congruence and $\boldsymbol{\mathcal { R }}_{i}$ contains exactly one line of $\mathcal{L}$, which we denote $\ell_{i}$. We choose $\boldsymbol{g}$ such that it preserves $\mathcal{L}$ and maps $\boldsymbol{\ell}_{2}$ to $\boldsymbol{\ell}_{1}$, and obtain $\boldsymbol{f}$ as $\boldsymbol{f}=\pi_{2} \circ \boldsymbol{g}^{-1}$.

In other words, given two cameras with same underlying congruence but distinct retinas, the image formed by one is projectively equivalent to the image formed by the other after some projective transformation of the space. Such a transformation is obviously nonunique.

\section{Linear congruences and admissible maps}

In this section, we present two representations of linear congruences: as linear sections of the Plücker quadric and as the integral lines of a projective map.

\subsection{Linear sections of the Plücker quadric}

Plücker coordinates are a classical parameterization of lines in $\mathbb{P}^{3}$ by the points of a quadric hypersurface $\mathbb{G}$ in $\mathbb{P}^{5}$, known under various names such as the Plücker quadric, the Klein quadric or the Grassmann manifold. For an introduction to Plücker coordinates we refer to [9], and simply mention some well-known facts we will use. The equation of the Plücker quadric is:

$$
(\mathbb{G}): \quad x_{0} x_{3}+x_{1} x_{4}+x_{2} x_{5}=0 .
$$

An affine line with Plücker coordinates $\left[x_{0}: \ldots: x_{5}\right]$ has direction $\left(x_{0}, x_{1}, x_{2}\right)^{T}$ and moment $\left(x_{3}, x_{4}, x_{5}\right)^{T}$ with respect to the origin. A point in $\mathbb{P}^{5} \backslash \mathbb{G}$ is called a screw. Let

$$
\langle x, y\rangle=\sum_{i=0}^{5} x_{i} y_{i} \text { and } \boldsymbol{x}^{*}=\left[x_{3}: x_{4}: x_{5}: x_{0}: x_{1}: x_{2}\right] \text {. }
$$

The incidence between two lines $\ell_{1}$ and $\ell_{2}$ writes as a bilinear relation between their Plücker coordinates $\boldsymbol{\xi}_{1}$ and $\boldsymbol{\xi}_{2}$ :

$$
\boldsymbol{\ell}_{1} \text { and } \boldsymbol{\ell}_{2} \text { intersect } \Longleftrightarrow\left\langle\boldsymbol{\xi}_{1}, \boldsymbol{\xi}_{2}^{*}\right\rangle=0 \text {. }
$$

The set of lines that meet a given, fixed, line is thus the intersection of the Plücker quadric with some hyperplane. Given a hyperplane $\boldsymbol{H}:\left\{\boldsymbol{x} \mid \sum_{i=0}^{5} h_{i} x_{i}=0\right\}$, let $\boldsymbol{h}$ denote the point $\left[h_{0}: \ldots: h_{5}\right]^{*}$. The point $\boldsymbol{h}$ lies on the quadric $\mathbb{G}$ if and only if $\boldsymbol{H}$ is tangent to $\mathbb{G}$ in $\boldsymbol{h}$. If $\boldsymbol{h}$ is a screw then the lines represented by $\boldsymbol{H} \cap \mathbb{G}$ are said to be conjugate to $\boldsymbol{h}$. In this setting, linear congruences can be defined, equivalently, as the two-dimensional sections of $\mathbb{G}$ by a projective space [11]. Specifically, line bundles and line fields are $\mathbb{P}^{2}$ 's

\begin{tabular}{|c|c|c|c|c|}
\hline $\operatorname{dim} \boldsymbol{H}$ & $\boldsymbol{H}^{\circ} \cap \mathbb{G}$ & type of $\boldsymbol{H} \cap \mathbb{G}$ & ambiguity locus & camera \\
\hline \hline \multirow{2}{*}{2} & & line field & $\mathbb{P}^{3}$ & \\
& & line bundle & a point & pinhole \\
\hline \multirow{3}{*}{3} & 2 points & hyperbolic & 2 skew lines & two-slit \\
& 1 point & parabolic & a line & pencil \\
& empty & elliptic & empty & oblique \\
& $\boldsymbol{H}^{\circ}$ & field + bundle & a plane & degenerate \\
\hline
\end{tabular}

Table 1: Linear congruences. We only count the real points of $\boldsymbol{H}^{\circ} \cap \mathbb{G}$ in the second column of the table.

contained in $\mathbb{G}$ and the remaining congruences (hyperbolic, parabolic, elliptic and degenerate) are sections of $\mathbb{G}$ by a $\mathbb{P}^{3}$. As a consequence, the Plücker coordinates of the lines of a linear camera are simply all possible linear combinations of the Plücker coordinates of 3 (for line bundles) or 4 (for other linear congruences) of its lines.

Given a subset $\boldsymbol{F}$ of $\mathbb{P}^{5}$, we denote by $\boldsymbol{F}^{\circ}$ its conjugate:

$$
\boldsymbol{F}^{\circ}=\left\{\boldsymbol{x} \in \mathbb{P}^{5} \mid \forall \boldsymbol{y} \in \boldsymbol{F},\left\langle\boldsymbol{x}, \boldsymbol{y}^{*}\right\rangle=0\right\} .
$$

Geometrically, if $\boldsymbol{F}$ is a family of lines and $\boldsymbol{H}$ is the span of $\boldsymbol{F}$ in $\mathbb{P}^{5}$, the line transversals to $\boldsymbol{F}$ are exactly the lines in $\boldsymbol{H}^{\circ} \cap \mathbb{G}$. In particular, if $\boldsymbol{H}$ is a three-dimensional projective subspace of $\mathbb{P}^{5}$ then $\boldsymbol{H}^{\circ}$ is a projective line of $\mathbb{P}^{5}$ whose intersection with the quadratic hypersurface $\mathbb{G}$ determines the type (hyperbolic, parabolic or elliptic) of the linear congruence $\boldsymbol{H} \cap \mathbb{G}$ (see Table 1).

Proposition 2. Two linear congruences have the same type if and only if they are projectively equivalent.

Proof. The statement is clear for line fields, line bundles and degenerate congruences so we consider only the remaining cases. If two linear congruences are projectively equivalent, so are their ambiguity loci and Table 1 shows that they have the same type. Conversely, consider two linear congruences $\mathcal{L}^{1}$ and $\mathcal{L}^{2}$, where $\mathcal{L}^{i}$ as all linear combinations of four pairwise skew and independant lines $\ell_{1}^{i}, \ldots, \ell_{4}^{i}$. There exists a map $f$ sending the triple $\left(\ell_{1}^{1}, \ldots, \ell_{3}^{1}\right)$ to the triple $\left(\ell_{1}^{2}, \ldots, \ell_{3}^{2}\right)$ [11, Proposition 3.1.13]. If $\mathcal{L}^{1}$ and $\mathcal{L}^{2}$ have the same type, the quadruples of lines have the same number of line transversals; thus, $f\left(\ell_{4}^{1}\right)$ and $\ell_{4}^{2}$ intersect the regulus generated by $\ell_{1}^{2}, \ldots, \ell_{3}^{2}$ in the same number of points. By Witt's Theorem [2, 13.7.1], there exists a projective transform $\boldsymbol{g}$ preserving the regulus that maps $\boldsymbol{f}\left(\boldsymbol{\ell}_{4}^{1}\right)$ to $\boldsymbol{\ell}_{4}^{2}$, and $\boldsymbol{g} \circ \boldsymbol{f}\left(\mathcal{L}^{1}\right)=\mathcal{L}^{2}$.

Corollary 3. Two reduced linear congruences have the same type if and only if they are projectively equivalent.

Proof. Two reduced linear congruences of distinct types have ambiguity loci of different nature and cannot be projectively equivalent. Conversely, two reduced linear congruences with same type come from linear congruences of identical types, which are projectively equivalent. 


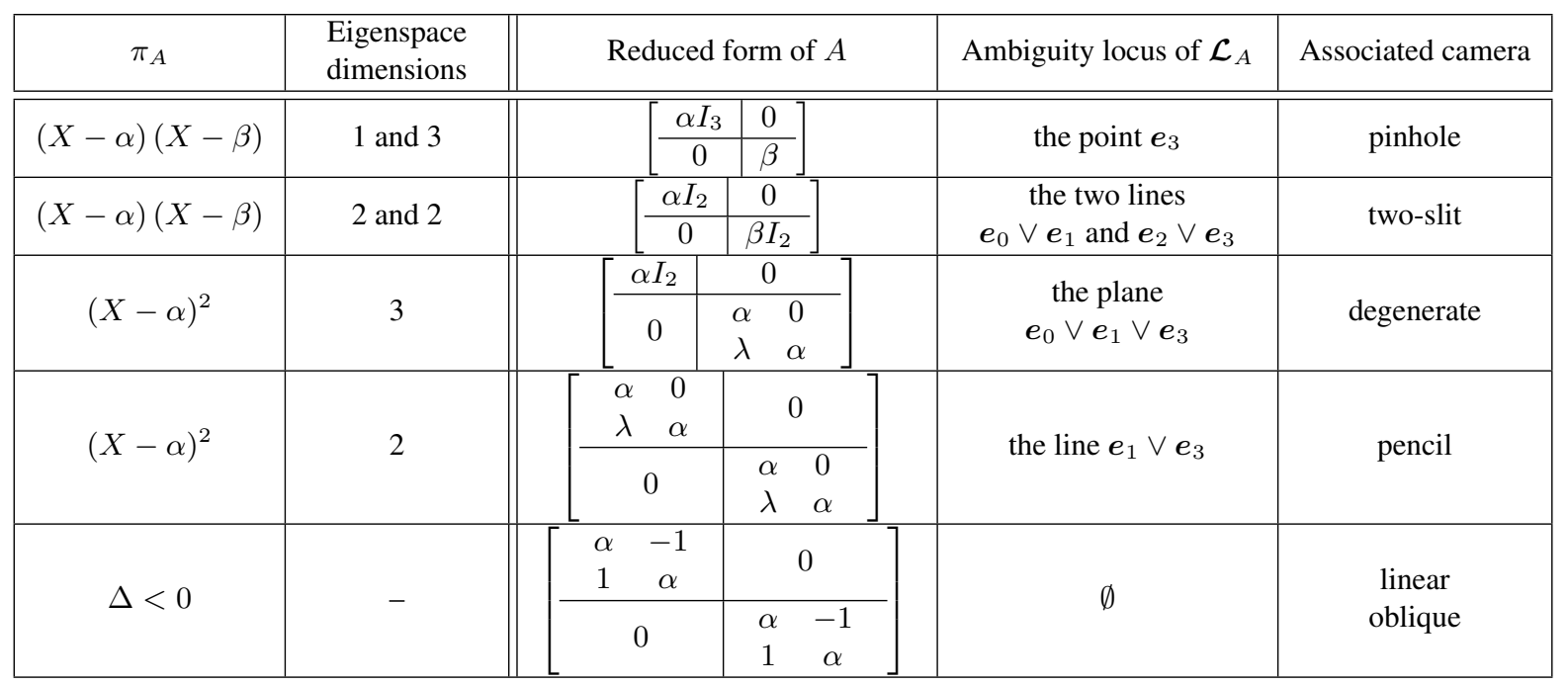

Table 2: Classification of admissible maps: for any admissible map $A$ there exists an invertible matrix $P$ such that $P A P^{-1}$ is one of the reduced form. Conventions: $\alpha \neq \beta, \lambda \neq 0$ and $\left(e_{0}, e_{1}, e_{2}, e_{3}\right)$ denotes the basis of $\mathbb{R}^{4}$ in which the reduced form of $A$ is written.

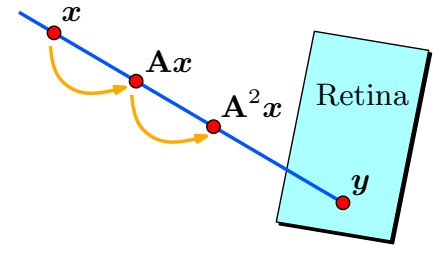

Figure 4: Linear cameras. The point $\mathbf{A}^{2} \boldsymbol{x}$ must lie on the line $\boldsymbol{x} \vee \mathbf{A} \boldsymbol{x}$ since otherwise two different lines $\left(\boldsymbol{x} \vee \mathbf{A} \boldsymbol{x}\right.$ and $\left.\mathbf{A} \boldsymbol{x} \vee \mathbf{A}^{2} \boldsymbol{x}\right)$ of the camera would pass through the point $\mathbf{A} \boldsymbol{x}$.

\subsection{Integral lines of an admissible map}

A matrix $A$ of size $4 \times 4$ can be identified with a linear map of $\mathbb{R}^{4}$ in its canonical basis, and also represents a projective map $\mathbf{A}$ of $\mathbb{P}^{3}$ in this basis. We associate with any such matrix $A$ the set of lines in $\mathbb{P}^{3}$

$$
\mathcal{L}_{A}=\{\boldsymbol{x} \vee \mathbf{A} \boldsymbol{x} \mid x \text { is not an eigenvector of } A\} .
$$

If $\mathcal{L}_{A}$ is a line congruence of order 1 then we say that $A$ is admissible and that $A$ induces $\mathcal{L}_{A}$, or that $\mathcal{L}_{A}$ is the set of integral lines of $A$. Recall that the minimal polynomial $\pi_{A}$ of a linear map $A$ is the unique monic polynomial of lowest degree such that $\pi_{A}(A)$ vanishes identically.

Proposition 4. A linear map $A: \mathbb{R}^{4} \rightarrow \mathbb{R}^{4}$ is admissible if and only if its minimal polynomial has degree 2 . The integral lines of an admissible map form a reduced linear congruence.

Sketch of proof. See Figure 4 for the intuition behind the first part of the result. The map $A$ induces a linear action $\Lambda^{2} A$ on the lines of $\mathbb{P}^{3}$ defined by $\boldsymbol{\Lambda}^{\mathbf{2}} \mathbf{A}(\boldsymbol{x} \vee \boldsymbol{y})=\mathbf{A} \boldsymbol{x} \vee \mathbf{A} \boldsymbol{y}$. We observe that $\mathcal{L}_{A}$ is contained in the kernel of $\Lambda^{2} A-$ $\pi_{A}(0) I_{6}$, which gives the intuition behind the linear nature of $\mathcal{L}_{A}$.
The eigenvalues of a map are precisely the roots of its minimal polynomial, so Proposition 4 implies that an admissible map $A$ has at most two real eigenvalues. Depending on their number and on the dimensions of the corresponding eigenspaces, a change of coordinates in $\mathbb{R}^{4}$ provides a matrix in one (and only one) of the reduced forms listed in Table 2. The ambiguity locus of a reduced linear congruence can be easily expressed in terms of the eigenspaces of an admissible map that represents it, as summarized in Table 2. Notice that every type of reduced linear congruence is present in Table 2. More generally:

Proposition 5. The sets of lines induced by admissible maps are exactly the reduced linear congruences.

Proof. By Proposition 4, any admissible map induces a reduced linear congruence. By Table 2, for any reduced linear congruence $\mathcal{L}$ there exists an admissible map $A$ such that $\mathcal{L}_{A}$ has the same type as $\mathcal{L}$. By Corollary 3 , there exists a change of coordinates in $\mathbb{R}^{4}$, with matrix $M$, that maps $\mathcal{L}_{A}$ to $\mathcal{L}$. The map $M^{-1} A M$ is clearly admissible, and a simple computation shows that it induces $\mathcal{L}$.

\section{Analytical projection formulas}

We now present direct and inverse projection formulas for linear cameras. In particular, we show that very simple formulas can be obtained when certain parameters of the cameras, which we call intrinsic parameters by analogy with the pinhole projection case, are known. This suggests introducing a new notion of normalized coordinates and internal calibration for linear cameras. 


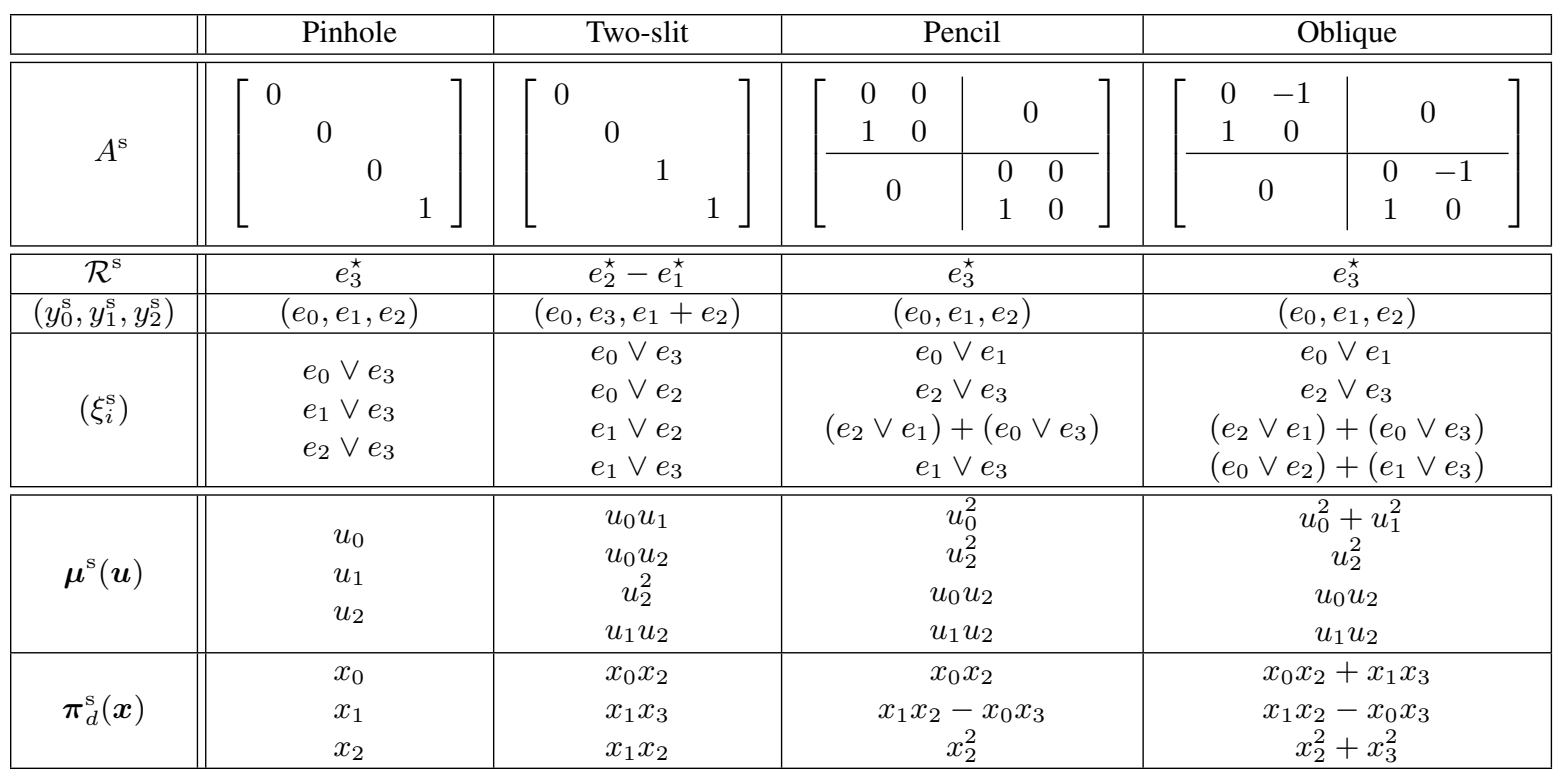

Table 3: Formulas for standard cameras. Here we denote by $\left(e_{0}, e_{1}, e_{2}, e_{3}\right)$ the canonical basis associated to $A^{o}$, and by $\left(e_{0}^{\star}, e_{1}^{\star}, e_{2}^{\star}, e_{3}^{\star}\right)$ the dual basis. Substituting $y_{0}^{\mathrm{s}}, y_{1}^{\mathrm{s}}, y_{2}^{\mathrm{s}}$ and $\mathcal{R}^{\mathrm{s}}$ for $y_{0}, y_{1}, y_{2}$ and $\mathcal{R}$ in Eqs. (2) and (3) gives the projection formulas given in this table.

\subsection{General formulas}

We first briefly rederive for completeness the general direct and inverse projection formulas for linear cameras given in [10]. Consider a linear camera whose reduced linear congruence $\mathcal{L}$ is induced by an admissible map $\mathbf{A}$ and whose retinal plane $\mathcal{R}$ is equipped with the basis $\left(\boldsymbol{y}_{0}, \boldsymbol{y}_{1}, \boldsymbol{y}_{2}\right)$. We identify $\mathcal{R}$ with its dual point. Let $\boldsymbol{x}$ be a non-ambiguous point in $\mathbb{P}^{3}, \xi$ the Plücker coordinates of the line $\ell$ of $\mathcal{L}$ through $\boldsymbol{x}$, and $\boldsymbol{y}$ the intersection point of $\boldsymbol{\ell}$ with $\mathcal{R}$. Let $\boldsymbol{u}$ denote the homogeneous coordinates of $\boldsymbol{y}$ in the basis of $\mathcal{R}$, so $y=\mathcal{Y} u$, where $\mathcal{Y}=\left[y_{0}, y_{1}, y_{2}\right]$. The direct projection is the map $\boldsymbol{\pi}_{d}: \boldsymbol{x} \mapsto \boldsymbol{u}$ and the inverse projection is the map $\boldsymbol{\pi}_{i}: \boldsymbol{u} \mapsto \boldsymbol{\xi}$

Since $\mathbf{A}$ induces $\mathcal{L}, \boldsymbol{\ell}=\boldsymbol{x} \vee \mathbf{A} \boldsymbol{x}$ and $\boldsymbol{y}$ is given by:

$$
\boldsymbol{y}=(\boldsymbol{x} \vee \mathbf{A} \boldsymbol{x}) \wedge \boldsymbol{\mathcal { R }}=\left((A x)^{T} \mathcal{R}\right) \boldsymbol{x}-\left(x^{T} \mathcal{R}\right) \mathbf{A} \boldsymbol{x} .
$$

The right part of Eq. (1) follows immediately from classical formulas [10] for the intersection of a line passing through two points with some plane. We can thus write the direct projection as a quadratic function of the coordinates of $\boldsymbol{x}$ :

$$
\mathcal{Y} \pi_{d}(x)=\left((A x)^{T} \mathcal{R}\right) x-\left(x^{T} \mathcal{R}\right) A x .
$$

As shown in [10], an explicit form for $\pi_{d}(x)$ is then easily obtained using a pseudoinverse of the matrix $\mathcal{Y}$, but this will not be necessary for the simplified equations obtained in the next section.

Now, let $\boldsymbol{\zeta}_{i j}=\boldsymbol{y}_{i} \vee \mathbf{A} \boldsymbol{y}_{j}+\boldsymbol{y}_{j} \vee \mathbf{A} \boldsymbol{y}_{i}$. Since $\boldsymbol{\xi}=\boldsymbol{y} \vee \mathbf{A} \boldsymbol{y}$ we have the following formula for inverse projection:

$$
\boldsymbol{\xi}=\sum_{0 \leq i \leq j \leq 2} u_{i} u_{j} \boldsymbol{\zeta}_{i j}
$$

Define $\widetilde{\mathcal{P}}=\left[\zeta_{00}, \zeta_{01}, \zeta_{02}, \zeta_{11}, \zeta_{12}, \zeta_{22}\right]$ and $\boldsymbol{\mu}: \mathbb{P}^{2} \rightarrow \mathbb{P}^{5}$ by:

$$
\boldsymbol{\mu}\left(\left[t_{0}: t_{1}: t_{2}\right]\right)=\left[t_{0}^{2}: t_{0} t_{1}: t_{0} t_{2}: t_{1}^{2}: t_{1} t_{2}: t_{2}^{2}\right] .
$$

The inverse projection rewrites as:

$$
\boldsymbol{\pi}_{i}(\boldsymbol{u})=\widetilde{\mathcal{P}} \boldsymbol{\mu}(\boldsymbol{u})
$$

\subsection{Formulas for standard cameras}

Ponce [10] observes that the lines of a linear congruence (in his terminology, a linear camera) can always be written as linear combinations of 4 or 5 lines or screws, and deduces inverse projection formulas with $6 \times 4$ or $6 \times 5$ matrices. In fact, since a linear congruence is the section of the Plücker quadric $\mathbb{G}$ by some $\mathbb{P}^{k}$ with $k=2$ or 3 (see Section 3.1), its lines are the linear combinations (on $\mathbb{G}$ ) of $k+1$ lines spanning that $\mathbb{P}^{k}$. We now show how this implies that all linear cameras admit inverse projection formulas with $6 \times$ 4 matrices. Notice that this does not immediately follows from Eq. (5) as a line $\boldsymbol{\zeta}_{i j}$ may not lie on $\boldsymbol{H}$.

We first consider four particular admissible maps, one per type of non-degenerate reduced linear congruence, obtained by setting $\alpha=0$ and $\beta=\lambda=1$ in the reduced forms of Table 2; they are the maps $A^{\mathrm{s}}$ in Table 3 . For each of these standard admissible maps, there is a natural choice of retina $\mathcal{R}^{\mathrm{s}}$ and of bases for both the retinal plane and the $\mathbb{P}^{k}$ meeting the Plücker quadric in $\mathcal{L}_{A^{\text {s }}}$ that yield simple, standard direct and inverse projection formulas. These choices are summarized in Table 3. Corollary 3 shows that for any linear camera there is one retinal plane and one basis for which the projections formulas are the standard ones (up to projective transformation). We now show that, in fact, for any retinal plane there are many bases for which this holds. 


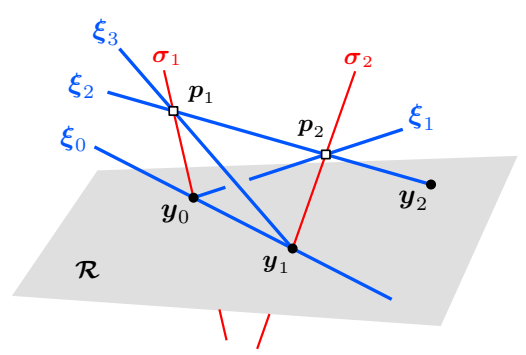

(a) Two-slit camera $\left(\boldsymbol{p}_{0}=\boldsymbol{y}_{0}, \boldsymbol{p}_{3}=\boldsymbol{y}_{1}\right.$ and $\left.y_{2}=p_{1}+p_{2}\right)$

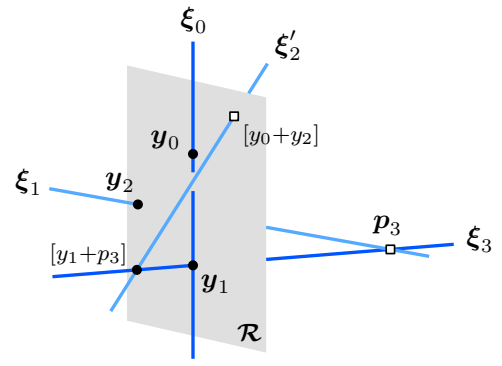

(b) Pencil camera $\left(\boldsymbol{p}_{i}=\boldsymbol{y}_{i}\right.$ for $\left.i=0,1,2\right)$.

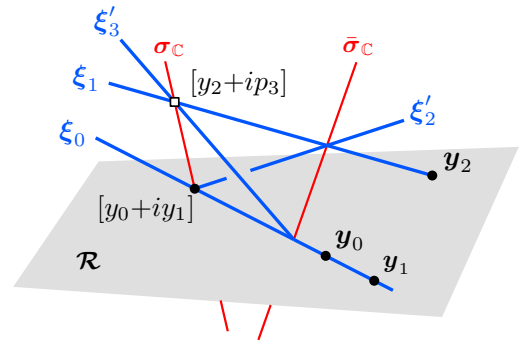

(c) Oblique camera $\left(\boldsymbol{p}_{i}=\boldsymbol{y}_{i}\right.$ for $\left.i=0,1,2\right)$.

Figure 5: Normalized coordinate systems for linear cameras.

\subsection{Formulas in normalized coordinates}

The simple formulas derived for standard cameras hold for any (generic) retinal plane whose basis capture certain geometric elements that characteristize the camera. By analogy with the pinhole case, we will call these elements the intrinsic parameters of the camera, and say that the coordinates of image points written in these bases are normalized. We exclude from our discussion pinhole cameras since any change of projective coordinate system for the retina will just amount to a linear change of coordinates and will thus not change the form of the projection equations. Let us define the intrinsic parameters and normalized coordinate systems associated with the three other types of cameras:

Two-slit cameras have two intrinsic parameters: the two intersection points of the retina with the slits. Any projective basis of the plane that contains these two points is normalized for the camera.

Pencil cameras have two intrinsic parameters: the intersection point of the retina with the axis of the pencil and the unique line of the camera contained in the retina. Any retinal basis that contains that point and another point on the line is normalized for the camera.

Oblique cameras have two intrinsic parameters: the real and imaginary parts of the complex intersection point of the retina with one of the two complex conjugate focal lines of the reduced linear congruence. The intersection point with the second line is simply the complex conjugate of this point, so it provides no supplemental intrinsic parameter. Any retinal basis that contains the real and imaginary parts of that point is normalized for the camera.

Notice that for each column in Table 3 , the retina basis is normalized since $\boldsymbol{y}_{0}^{\mathrm{s}}$ and $\boldsymbol{y}_{1}^{\mathrm{s}}$ meet the above conditions.

Proposition 6. The projection functions of a two-slit, pencil, or oblique camera, with reduced linear congruence $\mathcal{L}$, can be written in normalized coordinates as

$$
\boldsymbol{\pi}_{d}(\boldsymbol{x})=\boldsymbol{\pi}_{d}^{\mathrm{s}}(\mathbf{M} \boldsymbol{x}) \quad \text { and } \quad \boldsymbol{\xi}=\boldsymbol{\pi}_{i}(\boldsymbol{u})=\widetilde{\mathcal{P}}^{\mathrm{s}} \boldsymbol{\mu}^{\mathrm{s}}(\boldsymbol{u}),
$$

where $M$ is a change of coordinates, the lines/screws $\boldsymbol{\xi}_{i}$ are a basis of $\mathcal{L}, \widetilde{\mathcal{P}}^{\mathrm{s}}=\left[\boldsymbol{\xi}_{0}, \ldots, \boldsymbol{\xi}_{3}\right]$, and $\boldsymbol{\pi}_{d}^{\mathrm{s}}$ and $\boldsymbol{\mu}^{\mathrm{s}}$ are the functions from Table 3 for the associated type of camera.

Sketch of proof. For each type of linear camera, we construct $M=\left[p_{0}, \ldots, p_{3}\right]$ such that the family $\left(\xi_{i}\right)$ obtained by subsituting $p_{k}$ for $e_{k}$ in $\left(\xi_{i}^{\mathrm{s}}\right)$ given in Table 3 is a basis for the congruence of the linear camera. Figure 5 illustrates how to choose the $p_{i}$ 's. Thus $\mathbf{M}$ transforms $\mathcal{L}$ into the line congruence of the standard camera of the same type, and we can express the projection formulas as (6).

The analogy between our intrinsic parameters and normalized coordinates and those traditionally used for internally calibrated, Euclidean pinhole cameras is not strict: our "intrinsic parameters" are points/lines associated with the projective geometry of the camera instead of scalars associated with Euclidean characteristics. These points/lines (and thus, a normalized basis) are easily computed when the linear camera is given by an admissible map (via its reduced form) or a basis of four lines (via the conjugate to their span in Plücker space). It can also be computed from observed reference points using a ray-based calibration procedure for general non-central cameras $[5,13]$.

\section{Multi-view geometry for linear cameras}

Ponce [10] observes that the epipolar constraints for linear cameras can be written as a bilinear relation between quadratic forms of point coordinates measured in the retinal planes. In our setting, this is immediate: the epipolar constraint between two lines $\boldsymbol{\xi}$ and $\boldsymbol{\xi}^{\prime}$ of cameras $\mathcal{L}$ and $\mathcal{L}^{\prime}$ passing through the points with retinal coordinates $\boldsymbol{u}$ and $\boldsymbol{u}^{\prime}$ writes as $\left\langle\boldsymbol{\xi}, \boldsymbol{\xi}^{*}\right\rangle=0$ that is, with Eq. (5),

$$
\boldsymbol{\mu}(\boldsymbol{u})^{T} \mathcal{F} \boldsymbol{\mu}\left(\boldsymbol{u}^{\prime}\right)=0,
$$

where $\mathcal{F}=\widetilde{\mathcal{P}}^{T} \widetilde{\mathcal{P}}^{\prime *}$ is the $6 \times 6$ fundamental matrix associated with the two cameras and $\mu$ is given by Eq. (4). 


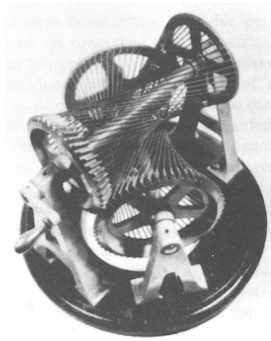

Figure 6: A physical realization of a pencil camera can be constructed by taking two hyperboloids of one sheet tangent to each other along one of their rulings, as in the gear system shown in this photograph. The tangents to both surfaces along this lines indeed form a parabolic linear congruence [11]. Image reproduced from [7, fig. 269].

Coordinate systems that lead to smaller inverse projection matrices also yield smaller fundamental matrices. Ponce [10] uses this fact to obtain $4 \times 4,4 \times 5$ and $5 \times 5$ fundamental matrices for pairs of non-central linear cameras. For internally calibrated linear cameras, Eq. (6) implies that the epipolar constraint write in normalized coordinates

$$
\boldsymbol{\mu}^{\mathrm{s}}(\boldsymbol{u})^{T} \mathcal{E} \boldsymbol{\mu}^{\mathrm{s}^{\prime}}\left(\boldsymbol{u}^{\prime}\right)=0,
$$

where $\mathcal{E}=\widetilde{\mathcal{P}}^{\mathrm{s} T} \widetilde{\mathcal{P}}^{\text {s } *}$ is the $4 \times 4$ essential matrix associated with the two cameras and $\mu^{\mathrm{s}}$ and $\mu^{\mathrm{s}^{\prime}}$ are the functions from Table 3 corresponding to the types of the cameras. Note that, although computing $\mathbf{M}$ in Eq. (6) requires knowing the position of the camera in some external projective basis of $\mathbb{P}^{3}$, we only need the intrinsic parameters to set up the normalized coordinate system and obtain the essential matrix formulation. In particular, when the intrinsic parameters are known, 15 correspondences suffice to compute the essential matrix $\mathcal{E}$ whereas 35 correspondences are needed for the fundamental matrix.

As shown in [10], the trifocal constraints associated with three linear cameras are captured by four trilinear constraints of the form

$$
\mathcal{T}_{j}\left(\pi_{i}(u), \pi_{i}^{\prime}\left(u^{\prime}\right), \pi_{i}^{\prime \prime}\left(u^{\prime \prime}\right)\right)=0 \quad \text { for } \quad j=1, \ldots, 4,
$$

where $\mathcal{T}_{j}$ is a $6 \times 6 \times 6$ tensor. Using Eq. (5) or Eq. (6) for uncalibrated or internally calibrated cameras respectively gives triquadratic expressions in $\boldsymbol{\mu}^{\mathrm{s}}(\boldsymbol{u}), \boldsymbol{\mu}^{\mathrm{s}^{\prime}}\left(\boldsymbol{u}^{\prime}\right)$, and $\boldsymbol{\mu}^{\mathrm{s}^{\prime \prime}}\left(\boldsymbol{u}^{\prime \prime}\right)$ associated with tensors of size $6 \times 6 \times 6$ or $4 \times 4 \times 4$.

\section{Discussion}

The general model for linear cameras we presented unifies the approaches of Ponce [10] and Pajdla [8] and affords simple formulas for direct and inverse projection as well as multi-view geometry using a new notion of intrinsic parameters and normalized coordinates. Our presentation has been theoretical, but we plan by the time of the conference to have implemented the estimation of the essential matrix from simulated, noisy image data. Next on our agenda is to construct a prototype of a new (to the best of our knowledge) physical model of pencil cameras (Figure 6), and conduct experiments with real data gathered with this proto- type. We also plan to investigate additional theoretical issues, such as the relationship between the essential matrix and the "extrinsic" parameters of the cameras involved, by analogy with the pinhole case.

\section{References}

[1] V. Benić and S. Gorjanc. (1,n) congruences. KOG, 10:5-12, 2006.

[2] M. Berger. Geometry II, volume 1 of Universitext. Springer, second edition, 1996.

[3] D. Feldman, T. Pajdla, and D. Weinshall. On the epipolar geometry of the crossed-slits projection. In Proc. ICCV, 2003.

[4] W. H. Greub. Linear Algebra. Springer, third edition, 1967.

[5] M. Grossberg and S. Nayar. The raxel imaging model and ray-based calibration. IJCV, 61(2):119137, 2005.

[6] R. Gupta and R. Hartley. Linear pushbroom cameras. IEEE Trans. PAMI, 19(9):963-975, 1997.

[7] D. Hilbert and S. Cohn-Vossen. Geometry and the Imagination. Chelsea Publishing Company, second edition, 1990.

[8] T. Pajdla. Stereo with oblique cameras. IJCV, 47(1):161-170, 2002.

[9] M. Pellegrini. Ray shooting and lines in space. In J. E. Goodman and J. O'Rourke, editors, Handbook of Discrete \& Computational Geometry, chapter 37, pages 839-856. CRC Press LLC, 2004.

[10] J. Ponce. What is a camera? In Proc. CVPR, pages 1526-1533, 2009.

[11] H. Pottmann and J. Wallner. Computational Line Geometry. Springer-Verlag, 2001.

[12] S. Seitz and J. Kim. The space of all stereo images. IJCV , 48(1):21-28, 2002.

[13] P. Sturm. Multi-view geometry for general camera models. In Proc. CVPR, pages 206-212, 2005.

[14] R. Sturm. Die Strahlencongruenzen erster und zweiter Ordnung. B. G. Teubner, 1893.

[15] O. Veblen and J. W. Young. Projective Geometry, volume 1. Blaisdell Publishing Co., 1910.

[16] J. Yu and L. McMillan. General linear cameras. In Proc. ECCV, 2004.

[17] J. Yu, L. McMillan, and P. Sturm. Multiperspective modeling, rendering, and imaging. In Proc. Eurographics, 2008. STAR - State of the Art Report.

[18] A. Zomet, D. Feldman, S. Peleg, and D. Weinshall. Mosaicing new views: The crossed-slits projection. IEEE Trans. PAMI, 25(6):741-754, 2003. 


\section{A. Equivalence of retinal planes}

This section is devoted to the proof of Proposition 1 from the paper. We begin with two lemmas.

Lemma 7. Consider a hyperbolic, elliptic or parabolic reduced linear congruence $\mathcal{L}$ and two lines $\ell_{1}$ and $\ell_{2}$ of that congruence. There is a projective transformation $g: \mathbb{P}^{3} \rightarrow$ $\mathbb{P}^{3}$ that preserving $\mathcal{L}$ globally and maps $\boldsymbol{\ell}_{1}$ to $\boldsymbol{\ell}_{2}$.

Proof. We first begin with general considerations. We suppose that $\ell_{1}$ and $\ell_{2}$ are skew (the proof adapts easily on the contrary). Given two skew lines $\ell$ and $\ell^{\prime}$ intersecting both $\ell_{1}$ and $\ell_{2}$, we define the points $\boldsymbol{e}_{0}, \ldots, \boldsymbol{e}_{3}$ as in Figure 7 .

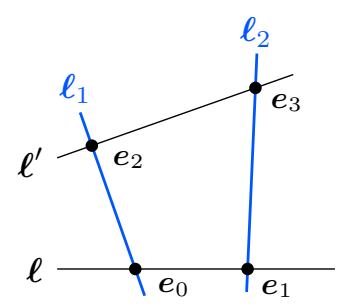

Figure 7: Definition of $e_{0}, \ldots, e_{3}$.

The family $\left(\boldsymbol{e}_{0}, \ldots, \boldsymbol{e}_{3}\right)$ forms a basis of $\mathbb{P}^{3}$. Consider now the map $\boldsymbol{g}$ whose matrix, written in this basis, is

$$
\operatorname{Mat}_{\left(e_{0}, e_{1}, e_{2}, e_{3}\right)} g=\left(\begin{array}{cc|cc}
0 & 1 & & \\
1 & 0 & & \\
\hline & & 0 & 1 \\
& & 1 & 0
\end{array}\right)
$$

The function $g$ clearly maps $\ell_{1}$ to $\ell_{2}$. For each type of congruence, we specify $\ell$ and $\ell^{\prime}$ and show that $g$ preserves $\mathcal{L}$ globally, which concludes the proof.

Hyperbolic congruence. Choosing $\ell$ and $\ell^{\prime}$ as the two focal lines of the congruence, the function $\boldsymbol{g}$ globally preserves the two focal lines, thus the congruence.

Elliptic congruence. Firstly, consider the situation over $\mathbb{C}$ and choose $\ell$ and $\ell^{\prime}$ as the two complex conjugate focal lines of the congruence. Notice that $\boldsymbol{e}_{1}=\overline{\boldsymbol{e}}_{0}$ and $\boldsymbol{e}_{3}=\overline{\boldsymbol{e}}_{2}$ and that $\boldsymbol{g}$ (as a function defined over $\mathbb{C}^{4}$ ) commutes with complex conjugaison. We can thus consider its restriction to the real space: we denote this restriction also by $\boldsymbol{g}$. It globally preserves the congruence since its complexification $\boldsymbol{g}_{\mathbb{C}}$ globally preserves $\ell$ and $\ell^{\prime}$ by definition.

Parabolic congruence. Choosing $\ell$ the focal line of the congruence, $\ell^{\prime}$ a line disjoint from $\ell$ and intersecting $\ell_{1}$ and $\ell_{2}$, Let us show that $g$ globally preserves the congruence. Consider the function $\boldsymbol{\nu}: \boldsymbol{\ell} \rightarrow \boldsymbol{\ell}^{\prime}$ that maps $\boldsymbol{x}$ to $\boldsymbol{y}$ where $\boldsymbol{x} \vee \boldsymbol{y}$ is the unique line of the congruence passing through $y$. For example, $\boldsymbol{\nu}\left(\boldsymbol{e}_{0}\right)=\boldsymbol{e}_{2}$ and $\boldsymbol{\nu}\left(\boldsymbol{e}_{1}\right)=\boldsymbol{e}_{3}$. According to [11, Theorem 3.2.9], $\nu$ is a projective transformation. Observe that $\nu$ entirely characterizes $\mathcal{L}$ : Indeed, the flat pencil of $\mathcal{L}$ with center $\boldsymbol{x} \in \boldsymbol{\ell}$ lies in the plane spanned by $\boldsymbol{\ell}$ and $\boldsymbol{\nu}(\boldsymbol{x})$. A straightforward computation shows that $\boldsymbol{g} \circ \boldsymbol{\nu}=\boldsymbol{\nu} \circ \boldsymbol{g}$ on $\ell$. It follows that $\boldsymbol{g}$ globally perserves $\mathcal{L}$.

Lemma $8([15, \S 107])$. Consider two linear cameras with the same reduced linear congruence $\mathcal{L}$ that is not a line bundle and with generic retinas $\boldsymbol{\mathcal { R }}_{i}(i=1,2)$. Let $\boldsymbol{\pi}_{i}$ be their projection functions, respectively. The restriction $\boldsymbol{\pi}_{2} \mid \mathcal{R}_{1}: \mathcal{R}_{1} \rightarrow \mathcal{R}_{2}$ is a projective transformation if and only if $\boldsymbol{\mathcal { R }}_{1}$ and $\boldsymbol{\mathcal { R }}_{2}$ share the same line of $\mathcal{L}$.

We give the proof of this lemma for the sake of completeness.

Proof. Denote by $\boldsymbol{\ell}_{i}$ the line of $\mathcal{L}$ contained in $\mathcal{R}_{i}$ and let $f=\left.\pi_{2}\right|_{\mathcal{R}_{1}}$. If $\ell_{1}$ and $\ell_{2}$ are distinct, $\ell_{1}$ intersects $\mathcal{R}_{2}$ in a point and so $\pi_{2}$ maps $\ell_{1}$ to that point: $f$ cannot be a projective transformation.

Conversely, suppose that $\ell_{1}=\ell_{2}=\ell$. We show that $f$ maps any line of $\boldsymbol{\mathcal { R }}_{1}$ to a line of $\boldsymbol{\mathcal { R }}_{2}$. The inverse map $f^{-1}=\left.\boldsymbol{\pi}_{1}\right|_{\mathcal{R}_{2}}$ has the same property by symetry. According to the Fundamental Theorem of Projective Geometry (see [11, Theorem 1.1.11] for example), $f$ is a projective transformation.

Let $\boldsymbol{d}$ be a line in $\mathcal{R}_{1}$ and let us show that $\boldsymbol{f}$ maps $\boldsymbol{d}$ to a line in $\mathcal{R}_{2}$. If $\boldsymbol{d}=\boldsymbol{\ell}$, this is clear, so assume that $\boldsymbol{d} \neq \boldsymbol{\ell}$. Except for at most two points, all the point of $\boldsymbol{d}$ are unambiguous. Consider two distinct (unambiguous) points $\boldsymbol{x}$ and $\boldsymbol{y}$ in $\boldsymbol{d}$ and not in $\boldsymbol{\ell}$. We denote by $\boldsymbol{\ell}_{\boldsymbol{x}}$ (resp. $\boldsymbol{\ell}_{\boldsymbol{y}}$ ) the unique line of $\mathcal{L}$ passing through $\boldsymbol{x}$ (resp. $\boldsymbol{y}$ ) and by $\mathscr{H}$ the regulus (possibly degenerate) generated by $\ell, \ell_{x}$ and $\ell_{y}$. All the rulings of $\mathscr{H}$ in the same family of $\left\{\boldsymbol{\ell}, \ell_{x}, \ell_{y}\right\}$ are contained in the linear congruence $\mathcal{L}$ because $\ell, \ell_{x}$ and $\ell_{\boldsymbol{y}}$ are. Thus, the image of $\boldsymbol{d}$ by $\boldsymbol{\pi}_{2}$ is exactly the intersection of $\mathscr{H}$ with $\boldsymbol{\mathcal { R }}_{2}$. This intersection is a pair of lines since it contains the lines $\ell$ (this intersection is clearly a pair of lines when $\mathscr{H}$ is degenerate). Denoting by $\boldsymbol{d}^{\prime}$ the other line of this intersection, it follows that $f$ maps $\boldsymbol{d}$ to $\boldsymbol{d}^{\prime}$ (see Figure 8).

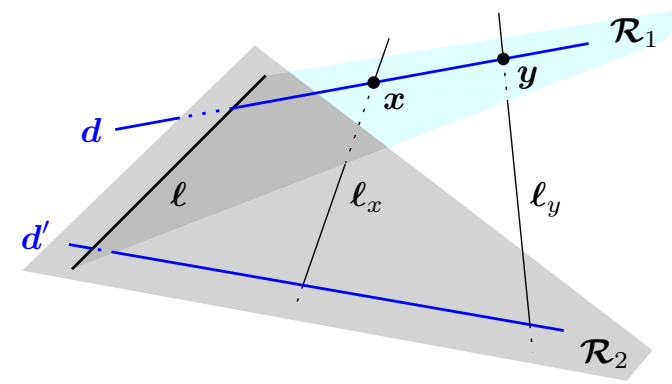

Figure 8: $\ell, \ell_{x}$ and $\ell_{y}$ are in $\mathcal{L}$ thus $f$ maps $d$ to $d^{\prime}$. 
We can now prove the equivalence of retinas given in Proposition 1 from the paper.

Proof. For a pinhole camera, the result immediately follows from the projective equivalence of central projection in $\mathbb{P}^{3}$. Consider a linear camera whose associated congruence $\mathcal{L}$ is hyperbolic, parabolic or elliptic. For $i=1,2$, we let $\boldsymbol{\mathcal { R }}_{i}$ be a generic retinal plane: $\ell_{i}$ denotes the unique line of $\mathcal{L}$ contained in $\boldsymbol{\mathcal { R }}_{i}$ and $\boldsymbol{\pi}_{i}$ the projection function for $\mathcal{L}$ on $\boldsymbol{\mathcal { R }}_{i}$. Let us show that $\boldsymbol{\pi}_{2}=\boldsymbol{f} \circ \boldsymbol{\pi}_{1} \circ \boldsymbol{g}$ with two projective transformations $\boldsymbol{f}: \mathcal{R}_{1} \rightarrow \mathcal{R}_{2}$ and $\boldsymbol{g}: \mathbb{P}^{3} \rightarrow \mathbb{P}^{3}$. By Lemma 7 , there is a projective transformation $\boldsymbol{g}: \mathbb{P}^{3} \rightarrow \mathbb{P}^{3}$ that preserves the set of lines of $\mathcal{L}$ and maps $\ell_{1}$ to $\ell_{2}$. The function $\tilde{\boldsymbol{\pi}}_{1}=\boldsymbol{g}^{-1} \circ \boldsymbol{\pi}_{1} \circ \boldsymbol{g}$ is thus a projection function for $\mathcal{L}$ on the retinal plane $\boldsymbol{g}^{-1}\left(\boldsymbol{\mathcal { R }}_{1}\right)$ containing $\boldsymbol{\ell}_{2}$. According to Lemma 8, we can choose $\boldsymbol{f}=\left.\boldsymbol{\pi}_{2}\right|_{\boldsymbol{g}^{-1}\left(\mathcal{R}_{1}\right)} \circ \boldsymbol{g}^{-1}$.

\section{B. Integral lines of a linear map}

We now give detailed proofs for the characterization and tabulation of admissible maps. This corresponds to Section 3.2 from the paper. In fact, we characterize and tabulate admissible maps in all dimensions.

Let $A$ denote a linear map $A: \mathbb{R}^{d+1} \rightarrow \mathbb{R}^{d+1}$, and assume that $d \geq 2$ (the case $d=1$ is trivial). We denote by $\mathcal{E}_{A}$ the union of the eigenspaces of $A$, by $\pi_{A}$ the minimal polynomial of $A$ and by $\Lambda^{2} A$ the linear action of $A$ on lines defined by:

$$
\forall x, y \in \mathbb{R}^{d+1} \quad\left(\Lambda^{2} A\right)(x \vee y)=A x \vee A y .
$$

When $A$ is an admissible map, we write $\mathcal{L}_{A}=\{\boldsymbol{x} \vee \boldsymbol{A x} \mid x \notin$ $\left.\mathcal{E}_{A}\right\}$. We will use the following lemma, which is a direct consequence of the theory of cyclic spaces in linear algebra (see [4, Chapter XIII $\S 3$ ] for example).

Lemma 9. Let $u$ be a linear map of $\mathbb{R}^{d}$ and $\pi_{u, x}$ the minimal polynomial of the principal ideal $\{P \in$ $\mathbb{R}[X] \mid P(u)(x)=0\}$. For almost all $x \in \mathbb{R}^{d}, \pi_{u, x}=\pi_{u}$.

Characterization. We first characterize admissible maps in terms of their minimal polynomials.

Lemma 10. $A$ is an admissible map if and only if $\pi_{A}$ has degree 2. If $A$ is an admissible map, the ambiguity locus of $\mathcal{L}_{A}$ is contained in $\mathcal{E}_{A}$.

Proof. For any $t \in \mathbb{R}$, we have that $A$ is an admissible map if and only if $A+t I_{d+1}$ is admissible, and that $\pi_{A}$ has degree 2 if and only if $\pi_{A+t I_{d+1}}$ has degree 2. It thus suffices to prove the statement when $A$ is invertible.

Assume that $A$ is nonsingular and admissible. For almost all points $\boldsymbol{x}$, neither $\boldsymbol{x}$ nor $\boldsymbol{A x}$ are in the ambiguity locus of $\mathcal{L}_{A}$. This implies that for almost all points $\boldsymbol{x}$, the lines $\boldsymbol{x} \vee \boldsymbol{A x}$ and $\boldsymbol{A x} \vee \boldsymbol{A}^{2} \boldsymbol{x}$ are the same and there is a linear relation between $x, A x$ and $A^{2} x$. This implies, with Lemma 9, that $\pi_{A}$ has degree at most 2. If $\pi_{A}$ has degree 1 or 0 then $A$ is trivially not admissible, so this proves the direct implication.

Conversely, assume that $A$ is nonsingular and that $\pi_{A}$ has degree 2. We write $\pi_{A}=X^{2}+\mu X+\nu$ where $\nu=\pi_{A}(0) \neq$ 0 as $A$ is nonsingular. Now, for any $x \notin \mathcal{E}_{A}$ we have

$$
\begin{aligned}
\left(\Lambda^{2} A\right)(x \vee A x) & =A x \vee A^{2} x \\
& =-A x \vee(\mu A x+\nu x) \\
& =\pi_{A}(0)(x \vee A x)
\end{aligned}
$$

Thus, $\boldsymbol{A}$ globally preserves all its integral lines. This means that if two lines of $\mathcal{L}_{A}$ pass through $\boldsymbol{x}$ then $\boldsymbol{x}$ is a fixed point under $\boldsymbol{A}$, and $x \in \mathcal{E}_{A}$. Since $\pi_{A}$ has degree exactly two, $\mathcal{E}_{A}$ is negligible and $A$ is admissible.

Classification. We now use the characterization of Lemma 10 to tabulate all admissible maps. In each case, we describe their set of integral lines, and in some case their ambiguity locus.

- If $\pi_{A}=(X-\alpha)(X-\beta), A$ has a reduced form of type $\left(A_{k}\right)$ with $1 \leq k \leq(d+1) / 2$

$$
A_{k}=\left(\begin{array}{c|c}
\alpha I_{k} & 0 \\
\hline 0 & \beta I_{d+1-k}
\end{array}\right)
$$

We denote by $\mathcal{E}_{\alpha}$ and $\mathcal{E}_{\beta}$ the eigenspaces of $A$ for the eigenvalues $\alpha$ and $\beta$ respectively.

- If $k=1$, we easily observe that the integral lines of $A$ form a line bundle with center $\mathcal{E}_{\alpha}$ : the ambiguity locus is reduced to a point.

- If $k>1$, we easily observe that the integral lines of $A$ are exactly those passing through a point of $\mathcal{E}_{\alpha}$ and through a point of $\mathcal{E}_{\beta}$. As these two sets are infinite, the ambiguity locus of $A$ is exactly $\mathcal{E}_{A}$, i.e. the union of two projective subspaces of complementary dimensions $k-1$ and $d-k$.

- If $\pi_{A}=(X-\alpha)^{2}, A$ has a reduced form of type $\left(B_{k}\right)$ with $1 \leq k \leq(d+1) / 2$

$$
\begin{gathered}
B_{k}=\left(\begin{array}{cccc}
U & & & \\
& \ddots & & \\
& & U & \\
& & & \alpha I_{d+1-2 k}
\end{array}\right) \\
\text { where } \quad U=\left(\begin{array}{cc}
\alpha & 0 \\
1 & \alpha
\end{array}\right)
\end{gathered}
$$


We have $\mathbb{R}^{d+1}=E_{1} \oplus E_{2} \oplus E_{3}$ with

$$
\begin{aligned}
& E_{1}=\operatorname{Vect}\left(e_{1}, e_{3}, \ldots, e_{2 k-1}\right) \\
& E_{2}=\operatorname{Vect}\left(e_{2 k}, \ldots, e_{d}\right) \\
& E_{3}=\operatorname{Vect}\left(e_{0}, e_{2}, \ldots, e_{2 k-2}\right)
\end{aligned}
$$

We can thus decompose a vector $x \notin \mathcal{E}_{A}$ into $x=$ $x_{1}+x_{2}+x_{3}$ with $x_{i} \in E_{i}$. Then,

$$
A x=\alpha x+\psi\left(x_{3}\right),
$$

where $\psi: E_{3} \rightarrow E_{1}$ is the map defined by $\psi\left(e_{2 i}\right)=$ $e_{2 i+1}$. The integral lines of $A$ are exactly those passing through $\boldsymbol{E}_{1}$ and not contained in $\mathcal{E}_{\alpha}$. The ambiguity locus of $\mathcal{L}_{A}$ is then exactly the projective subspace $\mathcal{E}_{A}$, of dimension $d-k$.

- If $\pi_{A}$ has no real root, $d+1$ is necessarly even and $A$ has a reduced form of type $(C)$ :

$$
C=\left(\begin{array}{ccc}
S & & \\
& \ddots & \\
& & S
\end{array}\right) \quad \text { where } S=\left(\begin{array}{cc}
\alpha & -1 \\
1 & \alpha
\end{array}\right)
$$

The ambiguity locus of $A$ is empty as $\mathcal{E}_{A}$ is empty.

To conclude, we note that the type $\left(A_{k}, B_{k}\right.$ or $\left.C\right)$ of an admissible map $A$ is entirely determined by the projective nature of the ambiguity locus of its integral lines $\mathcal{L}_{A}$.

\begin{tabular}{c|c} 
Type of $A$ & $\begin{array}{c}\text { Dim. of the components } \\
\text { of the ambiguity locus }\end{array}$ \\
\hline$\left(A_{1}\right)$ & 0 \\
$\left(A_{k}\right)$ & $k-1$ and $d-k$ \\
$\left(B_{k}\right)$ & $d-k$ \\
$(C)$ & -1
\end{tabular}

Table 4: Ambiguity loci of admissible maps $\left(1 \leq k \leq \frac{d+1}{2}\right)$.

Geometric interpretation. The next lemma, together with Lemma 10, proves Proposition 4 from the paper.

Lemma 11. If $\pi_{A}$ has degree 2 and $d \geq 3$, then $\mathcal{L}_{A}$ is a reduced linear congruence.

Proof. If $A$ is admissible then for any $t \in \mathbb{R}$ we have that $\mathcal{L}_{A}=\mathcal{L}_{A+t I_{d+1}}$. We can thus assume w.l.o.g. that $A$ is nonsingular. Define

$$
H_{A}=\operatorname{Ker}\left(\Lambda^{2} A-\pi_{A}(0) I_{d(d+1) / 2}\right),
$$

and observe that Equation (a) implies that $\mathcal{L}_{A} \subset \boldsymbol{H}_{A}$.
Let $\mathcal{L} \mathcal{E}_{A}$ denote the set of lines contained in $\mathcal{E}_{A}$. Let $\boldsymbol{x} \vee$ $\boldsymbol{y} \in \boldsymbol{H}_{A} \cap \mathbb{G}_{d}$ (where $\mathbb{G}_{d}$ is the Grassmannian representing the lines of $\mathbb{P}^{d}$ ). By definition of $H_{A}$, we have:

$$
\Lambda^{2} A(x \vee y)=A x \vee A y=\pi_{A}(0)(x \vee y) .
$$

We distinguish between three cases.

- If $x \notin \mathcal{E}_{A}$ then Equality (b) yields that $\boldsymbol{A x} \in \boldsymbol{x} \vee \boldsymbol{y}$, and we have that $\boldsymbol{x} \vee \boldsymbol{y}=\boldsymbol{x} \vee A \boldsymbol{x}$. A symmetric argument holds if $y \notin \mathcal{E}_{A}$.

- If $x$ and $y$ are two eigenvectors of $A$ with distinct eigenvalues $\lambda_{x} \neq \lambda_{y}$ then $z=x+y$ is not an eigenvector of $A$, and

$$
z \vee A z=x \vee A y+y \vee A x=\left(\lambda_{y}-\lambda_{x}\right)(x \vee y)
$$

yields that the line $\boldsymbol{x} \vee \boldsymbol{y}$ is the same as $\boldsymbol{z} \vee A \boldsymbol{z}$.

- If $x$ and $y$ are two noncolinear eigenvectors with the same eigenvalue $\lambda$, the line $\boldsymbol{x} \vee \boldsymbol{y}$ is contained in $\mathcal{E}_{A}$. In that case, $\Lambda^{2} A(x \vee y)=\lambda^{2}(x \vee y)$, and Equation (b) implies that $\pi_{A}(0)=\lambda^{2}$. It follows that $\lambda$ is a double root of $\pi_{A}$.

Altogether, this proves that

$$
\boldsymbol{H}_{A} \cap \mathbb{G}_{d}=\left\{\begin{array}{cl}
\mathcal{L}_{A} & \text { if } \pi_{A} \text { has no double root } \\
\mathcal{L}_{A} \cup \mathcal{L} \mathcal{E}_{A} & \text { if } \pi_{A} \text { has a double root }
\end{array} .\right.
$$

In the case where $\pi_{A}$ has no mutiple root, this proves that $\mathcal{L}_{A}$ is a line congruence, no line of which is contained in $\mathcal{E}_{A}$; by Lemma 10 , this guarantees that $\mathcal{L}_{A}$ is also a reduced line congruence. If $\pi_{A}$ has a double root, the above classification shows that the reduced form of $A$ is of type $\left(B_{k}\right)$ and the ambiguity locus of $\mathcal{L}_{A}$ is exactly $\mathcal{E}_{A}$. Thus, the ambiguity locus of $\boldsymbol{H}_{A} \cap \mathbb{G}_{d}=\mathcal{L}_{A} \cup \mathcal{L} \mathcal{E}_{A}$ is included in $\mathcal{E}_{A}$. If $\operatorname{dim} \mathcal{E}_{A} \geq 2$, the ambiguity locus of $\boldsymbol{H}_{A} \cap \mathbb{G}_{d}$ is $\mathcal{E}_{A}$. If $\operatorname{dim} \mathcal{E}_{A}=1$, we have $d=3$ and $k=2$. The previous classification then implies that there is a infinite number of lines of $\mathcal{L}_{A}$ passing through any point of $\mathcal{E}_{A}$, so the ambiguity locus of $\boldsymbol{H}_{A} \cap \mathbb{G}_{d}$ is exactly $\mathcal{E}_{A}$. As a consequence, the reduced linear congruence obtained from $\boldsymbol{H}_{A} \cap \mathbb{G}_{d}$ is exactly $\mathcal{L}_{A}$.

\section{Standard cameras}

In this section, we detail for each type of camera all computations that lead to the formulas of $\boldsymbol{\mu}^{\mathrm{s}}(\boldsymbol{u})$ and $\boldsymbol{\pi}_{d}^{\mathrm{s}}(\boldsymbol{x})$ of Table 3 from the paper (reproduced for convenience as Table B). It simply consists of substituting the expressions of $A^{\mathrm{s}}$ and $\mathcal{R}^{\mathrm{s}}$ in Eq (1) and (3) from the paper. We also show that any normalized basis of a generic retina provides the standard formulas of Table B, hence proving Proposition 6 . The results for pinhole cameras are straightforward. 


\begin{tabular}{|c|c|c|c|c|c|c|}
\hline & Pinhole & Two-slit & \multicolumn{2}{|c|}{ Pencil } & \multicolumn{2}{|c|}{ Oblique } \\
\hline & $\begin{array}{ll}0 \\
& \\
& \end{array}$ & {$\left[\begin{array}{ll}0 & \\
& 0\end{array}\right]$} & $\begin{array}{ll}0 & 0 \\
1 & 0\end{array}$ & 0 & $\begin{array}{cc}0 & -1 \\
1 & 0\end{array}$ & 0 \\
\hline$A^{\circ}$ & $\begin{array}{ll}0 & \\
& 1\end{array}$ & 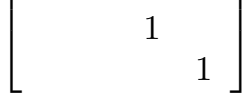 & U & $\begin{array}{ll}0 & 0 \\
1 & 0\end{array}$ & 0 & $\begin{array}{cc}0 & -1 \\
1 & 0\end{array}$ \\
\hline $\mathcal{R}^{\mathrm{S}}$ & $e_{3}^{\star}$ & $e_{2}^{\star}-e_{1}^{\star}$ & & & & \\
\hline$\left(y_{0}^{\mathrm{s}}, y_{1}^{\mathrm{s}}, y_{2}^{\mathrm{s}}\right)$ & $\left(e_{0}, e_{1}, e_{2}\right)$ & $\left(e_{0}, e_{3}, e_{1}+e_{2}\right)$ & $\left(e_{0}\right.$ &,$\left.e_{2}\right)$ & $\left(e_{0}\right.$, &,$\left.e_{2}\right)$ \\
\hline$\left(\xi_{i}^{\mathrm{S}}\right)$ & $\begin{array}{l}e_{0} \vee e_{3} \\
e_{1} \vee e_{3} \\
e_{2} \vee e_{3}\end{array}$ & $\begin{array}{l}e_{0} \vee e_{3} \\
e_{0} \vee e_{2} \\
e_{1} \vee e_{2} \\
e_{1} \vee e_{3} \\
\end{array}$ & $\begin{array}{r}e_{0} \\
e_{2} \\
\left(e_{2} \vee e_{1}\right) \\
e_{1}\end{array}$ & $\begin{array}{l}e_{1} \\
e_{3} \\
\left(e_{0} \vee e_{3}\right) \\
e_{3}\end{array}$ & $\begin{array}{r}e_{0} \\
e_{2} \\
\left(e_{2} \vee e_{1}\right) \\
\left(e_{0} \vee e_{2}\right) \\
\end{array}$ & $\begin{array}{l}e_{1} \\
e_{3} \\
\left(e_{0} \vee e_{3}\right) \\
\left(e_{1} \vee e_{3}\right)\end{array}$ \\
\hline $\boldsymbol{\mu}^{\mathrm{s}}(\boldsymbol{u})$ & $\begin{array}{l}u_{0} \\
u_{1} \\
u_{2}\end{array}$ & $\begin{array}{c}u_{0} u_{1} \\
u_{0} u_{2} \\
u_{2}^{2} \\
u_{1} u_{2}\end{array}$ & & & & $\begin{array}{l}u_{1}^{2} \\
2 \\
2\end{array}$ \\
\hline $\boldsymbol{\pi}_{d}^{\mathrm{s}}(\boldsymbol{x})$ & $\begin{array}{l}x_{0} \\
x_{1} \\
x_{2}\end{array}$ & $\begin{array}{l}x_{0} x_{2} \\
x_{1} x_{3} \\
x_{1} x_{2}\end{array}$ & $x_{1} x_{2}$ & $x_{0} x_{3}$ & $\begin{array}{r}x_{0} x_{2} \\
x_{1} x_{2} \\
x_{2}^{2}\end{array}$ & $\begin{array}{l}x_{1} x_{3} \\
x_{0} x_{3} \\
x_{3}^{2}\end{array}$ \\
\hline
\end{tabular}

Table 5: Formulas for standard cameras. Here we denote by $\left(e_{0}, e_{1}, e_{2}, e_{3}\right)$ the canonical basis associated to $A^{o}$, and by $\left(e_{0}^{\star}, e_{1}^{\star}, e_{2}^{\star}, e_{3}^{\star}\right)$ the dual basis.

Two-slit camera. Consider the standard two-slit camera induced by the admissible map

$$
A^{\mathrm{s}}=\left(\begin{array}{cccc}
0 & & & \\
& 0 & & \\
& & 1 & \\
& & & 1
\end{array}\right)
$$

and whose retina $\mathcal{R}^{\mathrm{s}}$ has basis $\left(e_{0}, e_{3}, e_{1}+e_{2}\right)$. The retina is represented by the dual point $\left(e_{2}-e_{1}\right)^{\star}$. The image point $\boldsymbol{y} \in \mathcal{R}$ of $\boldsymbol{x}$ is given by

$$
\begin{aligned}
y & =\left(x \vee A^{\mathrm{s}} x\right) \wedge\left(e_{2}-e_{1}\right) \\
& =\left(\left(A^{\mathrm{s}} x\right)^{T}\left(e_{2}-e_{1}\right)\right) x-\left(x^{T}\left(e_{2}-e_{1}\right)\right) A^{\mathrm{s}} x \\
& =x_{2} x+\left(x_{1}-x_{2}\right) A^{\mathrm{s}} x=\left(\begin{array}{c}
x_{0} x_{2} \\
x_{1} x_{2} \\
x_{1} x_{2} \\
x_{1} x_{3}
\end{array}\right)
\end{aligned}
$$

We directly observe in this expression that the coordinates of $\boldsymbol{y}$ in the standard basis of $\boldsymbol{\mathcal { R }}$ are $\boldsymbol{u}=\left[x_{0} x_{2}: x_{1} x_{3}\right.$ : $\left.x_{1} x_{2}\right]$ (the second and third coordinates of $\boldsymbol{y}$ are equal). This establishes the expression of $\boldsymbol{\pi}_{d}^{\mathrm{s}}$.

We now compute the inverse projection $\boldsymbol{\pi}_{i}^{\mathrm{s}}: \boldsymbol{u} \mapsto \boldsymbol{\xi}$ of the standard two-slit camera. Start with

$$
A^{\mathrm{s}} y=\left(\begin{array}{cccc}
0 & & & \\
& 0 & & \\
& & 1 & \\
& & & 1
\end{array}\right) \cdot\left(\begin{array}{c}
u_{0} \\
u_{2} \\
u_{2} \\
u_{1}
\end{array}\right)=\left(\begin{array}{c}
0 \\
0 \\
u_{2} \\
u_{1}
\end{array}\right)
$$

and now:

$$
\begin{aligned}
\xi= & y \vee A^{\mathrm{s}} y \\
= & \left(u_{0} e_{0}+u_{2} e_{1}+u_{2} e_{2}+u_{1} e_{3}\right) \vee\left(u_{2} e_{2}+u_{1} e_{3}\right) \\
= & u_{0} u_{1}\left(e_{0} \vee e_{3}\right) \\
& +u_{0} u_{2}\left(e_{0} \vee e_{2}\right) \\
& +u_{2}^{2}\left(e_{1} \vee e_{2}\right) \\
& +u_{1} u_{2}\left(e_{1} \vee e_{3}\right)
\end{aligned}
$$

We obtain the expression of $\mu^{\mathrm{s}}(u)$.

Let us prove Proposition 6 for two-slit cameras. Consider a two-slit camera whose linear congruence is denoted by $\mathcal{L}$ and whose retina $\mathcal{R}$ is endowed with a normalized basis

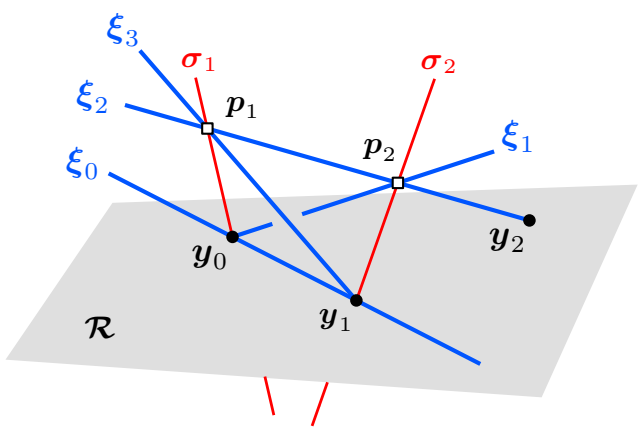

Figure 9: Two-slit camera $\left(\boldsymbol{p}_{0}=\boldsymbol{y}_{0}, \boldsymbol{p}_{3}=\boldsymbol{y}_{1}\right.$ and $y_{2}=$ $\left.p_{1}+p_{2}\right)$. 
$\left(\boldsymbol{y}_{0}, \boldsymbol{y}_{1}, \boldsymbol{y}_{2}\right)$. Let us define the points $\boldsymbol{p}_{i}$ as follows. We let $p_{0}=y_{0}$ and $p_{3}=y_{1}$. Since $\mathcal{R}$ intersects the ambiguity locus of $\mathcal{L}$ in precisely $\left\{\boldsymbol{p}_{0}, \boldsymbol{p}_{3}\right\}$ there is a unique line $\boldsymbol{\ell}^{\prime}$ of $\mathcal{L}$ through $\boldsymbol{y}_{1}$. Denoting the two slits of the camera by $\boldsymbol{\sigma}_{1}$ and $\boldsymbol{\sigma}_{2}$, we let $\boldsymbol{p}_{i}=\boldsymbol{\ell} \cap \boldsymbol{\sigma}_{i}$ for $i=1,2$ such that $y_{2}=$ $p_{1}+p_{2}$. Now we consider the following basis on $\mathcal{L}$ :

$$
\begin{array}{ll}
\xi_{0}=p_{0} \vee p_{3}, & \xi_{1}=p_{0} \vee p_{2}, \\
\xi_{2}=p_{1} \vee p_{2}, & \xi_{3}=p_{1} \vee p_{3} .
\end{array}
$$

Letting $M=\left[p_{0}, \ldots, p_{3}\right]$ and $\tilde{\boldsymbol{x}}=M \boldsymbol{x}$, we get that $\mathbf{M}$ maps $\mathcal{L}$ to the linear congruence of the standard camera (the basis $\left(\xi_{i}\right)$ is mapped to $\left(\xi_{i}^{\mathrm{s}}\right)$ ) and maps $\mathcal{R}$ to $\mathcal{R}^{\mathrm{s}}$ (the basis $\left(y_{i}\right)$ is mapped to $\left.\left(y_{i}^{\mathrm{s}}\right)\right)$. Up to projective transformation, the projection formulas are the standard one:

$$
\boldsymbol{u}=\left[\begin{array}{c}
\tilde{x}_{0} \tilde{x}_{2} \\
\tilde{x}_{1} \tilde{x}_{3} \\
\tilde{x}_{1} \tilde{x}_{2}
\end{array}\right] \quad \text { and } \quad \boldsymbol{\xi}=\left[\xi_{1}, \ldots, \xi_{4}\right]\left[\begin{array}{c}
u_{0} u_{1} \\
u_{0} u_{2} \\
u_{2}^{2} \\
u_{1} u_{2}
\end{array}\right]
$$

and Proposition 6 for two-slit cameras follows.

Pencil camera. Consider the standard pencil camera induced by the admissible map

$$
A^{\mathrm{s}}=\left(\begin{array}{cc|cc}
0 & 0 & & 0 \\
1 & 0 & & \\
\hline 0 & 0 & 0 \\
& 1 & 0
\end{array}\right)
$$

and whose retina $\mathcal{R}^{\mathrm{s}}$ has basis $\left(e_{0}, e_{1}, e_{2}\right)$. The retina is represented by the dual point $e_{3}^{\star}$. The image point $\boldsymbol{y} \in \mathcal{R}$ of $\boldsymbol{x}$ is given by

$$
\begin{aligned}
y & =\left(x \vee A^{\mathrm{s}} x\right) \wedge e_{3}=\left(\left(A^{\mathrm{s}} x\right)^{T} e_{3}\right) x-\left(x \cdot e_{3}\right) A^{\mathrm{s}} x \\
& =x_{2} x-x_{3} A^{\mathrm{s}} x=-\left(\begin{array}{c}
x_{0} x_{2} \\
x_{1} x_{2}-x_{0} x_{3} \\
x_{2}^{2} \\
0
\end{array}\right)
\end{aligned}
$$

We directly deduce the coordinates of $\boldsymbol{y}$ in the standard basis of $\boldsymbol{\mathcal { R }}^{\mathrm{s}}$ and we establish the expression of $\boldsymbol{\pi}_{d}^{\mathrm{s}}$.

We now compute the inverse projection $\boldsymbol{\pi}_{i}^{\mathrm{s}}: \boldsymbol{u} \mapsto \boldsymbol{\xi}$ of the standard pencil camera. Start with

$$
A^{\mathrm{s}} y=\left(\begin{array}{cc|cc}
0 & 0 & \multicolumn{1}{c}{0} \\
1 & 0 & & \\
\hline 0 & 0 & 0 \\
0 & 1 & 0
\end{array}\right) \cdot\left(\begin{array}{c}
u_{0} \\
u_{1} \\
u_{2} \\
0
\end{array}\right)=\left(\begin{array}{c}
0 \\
u_{0} \\
0 \\
u_{2}
\end{array}\right)
$$

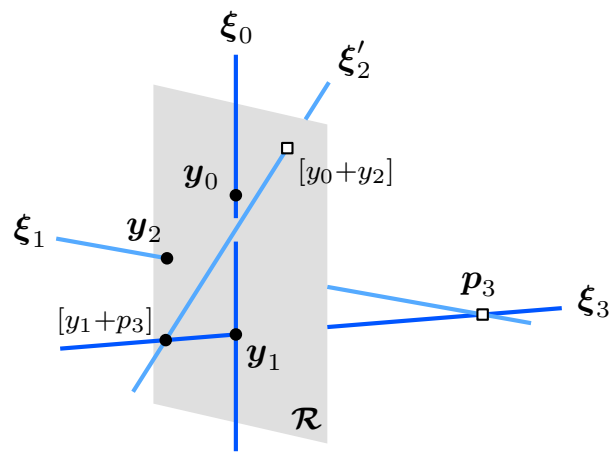

Figure 10: Pencil camera $\left(\boldsymbol{p}_{i}=\boldsymbol{y}_{i}\right.$ for $\left.i=0,1,2\right)$.

and now:

$$
\begin{aligned}
\xi= & y \vee A^{\mathrm{s}} y \\
= & \left(u_{0} e_{0}+u_{1} e_{1}+u_{2} e_{2}\right) \vee\left(u_{0} e_{1}+u_{2} e_{3}\right) \\
= & u_{0}^{2}\left(e_{0} \vee e_{1}\right) \\
& +u_{2}^{2}\left(e_{2} \vee e_{3}\right) \\
& +u_{0} u_{2}\left(\left(e_{0} \vee e_{3}\right)+\left(e_{2} \vee e_{1}\right)\right) \\
& +u_{1} u_{2}\left(e_{1} \vee e_{3}\right)
\end{aligned}
$$

We obtain the expression of $\mu^{\mathrm{s}}(u)$.

Let us prove Proposition 6 for pencil cameras. Consider a pencil camera whose linear congruence is denoted by $\mathcal{L}$ and whose retina $\mathcal{R}$ is endowed with a normalized basis $\left(\boldsymbol{y}_{0}, \boldsymbol{y}_{1}, \boldsymbol{y}_{2}\right)$. Let us define the points $\boldsymbol{p}_{i}$ as follows. We let $p_{i}=y_{i}$ for $i=1,2,3$. Since $\mathcal{R}$ intersects the ambiguity locus of $\mathcal{L}$ in precisely $\left\{\boldsymbol{p}_{1}\right\}$, there is a unique line $\ell^{\prime}$ of $\mathcal{L}$ through $\boldsymbol{p}_{2}$. Now we can choose $p_{3}$ such that $\xi_{2}^{\prime}=\left(p_{0}+\right.$ $\left.p_{2}\right) \vee\left(p_{1}+p_{3}\right) \in \mathcal{L}$ and consider the following basis on $\mathcal{L}$ :

$$
\begin{array}{ll}
\xi_{0}=p_{0} \vee p_{1}, & \xi_{1}=p_{2} \vee p_{3}, \\
\xi_{2}^{\prime}=\left(p_{0}+p_{2}\right) \vee\left(p_{1}+p_{3}\right), & \xi_{3}=p_{1} \vee p_{3} .
\end{array}
$$

As $\xi_{2}=\left(p_{2} \vee p_{1}\right)+\left(p_{0} \vee p_{3}\right)$ is a linear combination of the $\xi_{0}, \xi_{1}, \xi_{2}^{\prime}, \xi_{3}$, the family $\left(\xi_{i}\right)$ is a basis of $\mathcal{L}$. Letting $M=\left[p_{0}, \ldots, p_{3}\right]$ and $\tilde{\boldsymbol{x}}=M \boldsymbol{x}$, we get that $\mathbf{M}$ maps $\mathcal{L}$ to the linear congruence of the standard camera (the basis $\left(\xi_{i}\right)$ is mapped to $\left(\xi_{i}^{\mathrm{s}}\right)$ ) and maps $\mathcal{R}$ to $\boldsymbol{\mathcal { R }}^{\mathrm{s}}$ (the basis $\left(y_{i}\right)$ is mapped to $\left.\left(y_{i}^{\mathrm{s}}\right)\right)$. Up to projective transformation, the projection formulas are the standard one:

$$
\boldsymbol{u}=\left[\begin{array}{c}
\tilde{x}_{0} \tilde{x}_{2} \\
\tilde{x}_{1} \tilde{x}_{2}-\tilde{x}_{0} \tilde{x}_{3} \\
\tilde{x}_{2}^{2}
\end{array}\right] \text { and } \boldsymbol{\xi}=\left[\xi_{1}, \ldots, \xi_{4}\right]\left[\begin{array}{c}
u_{0}^{2} \\
u_{2}^{2} \\
u_{0} u_{2} \\
u_{1} u_{2}
\end{array}\right]
$$

and Proposition 6 for pencil cameras follows. 


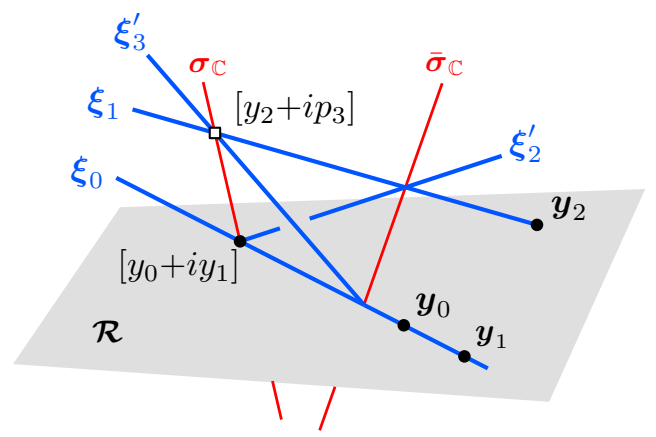

Figure 11: Oblique camera $\left(\boldsymbol{p}_{i}=\boldsymbol{y}_{i}\right.$ for $\left.i=0,1,2\right)$.

Oblique camera. Consider the standard oblique camera induced by the admissible map

$$
A^{\mathrm{s}}=\left(\begin{array}{cc|cc}
0 & -1 & & 0 \\
1 & 0 & & \\
\hline 0 & 0 & -1 \\
0 & 1 & 0
\end{array}\right) \cdot\left(\begin{array}{c}
u_{0} \\
u_{1} \\
u_{2} \\
0
\end{array}\right)
$$

and whose retina $\mathcal{R}^{\mathrm{s}}$ has basis $\left(e_{0}, e_{1}, e_{2}\right)$. The retina is represented by the dual point $e_{3}^{\star}$. The image point $\boldsymbol{y} \in \mathcal{R}$ of $\boldsymbol{x}$ is given by

$$
\begin{aligned}
y & =\left(x \vee A^{\mathrm{s}} x\right) \wedge e_{3}=\left(\left(A^{\mathrm{s}} x\right)^{T} e_{3}\right) x-\left(x \cdot e_{3}\right) A^{\mathrm{s}} x \\
& =x_{2} x-x_{3} A^{\mathrm{s}} x=-\left(\begin{array}{c}
x_{0} x_{2}+x_{1} x_{3} \\
x_{1} x_{2}-x_{0} x_{3} \\
x_{2}^{2}+x_{3}^{2} \\
0
\end{array}\right)
\end{aligned}
$$

We directly deduce the coordinates of $\boldsymbol{y}$ in the standard basis of $\boldsymbol{\mathcal { R }}^{\mathrm{s}}$ and we establish the expression of $\boldsymbol{\pi}_{d}^{\mathrm{s}}$.

We now compute the inverse projection $\boldsymbol{\pi}_{i}^{\mathrm{s}}: \boldsymbol{u} \mapsto \boldsymbol{\xi}$ of the standard oblique camera. Start with

$$
A^{\mathrm{s}} y=\left(\begin{array}{cc|cc}
0 & -1 & & 0 \\
1 & 0 & & \\
\hline 0 & 0 & -1 \\
& 0 & 0
\end{array}\right) \cdot\left(\begin{array}{c}
u_{0} \\
u_{1} \\
u_{2} \\
0
\end{array}\right)=\left(\begin{array}{c}
-u_{1} \\
u_{0} \\
0 \\
u_{2}
\end{array}\right)
$$

and now:

$$
\begin{aligned}
\xi= & y \vee A^{\mathrm{s}} y \\
= & \left(u_{0} e_{0}+u_{1} e_{1}+u_{2} e_{2}\right) \vee\left(-u_{1} e_{0}+u_{0} e_{1}+u_{2} e_{3}\right) \\
= & \left(u_{0}^{2}+u_{1}^{2}\right)\left(e_{0} \vee e_{1}\right)+u_{2}^{2}\left(e_{2} \vee e_{3}\right) \\
& +u_{0} u_{2}\left(\left(e_{0} \vee e_{3}\right)+\left(e_{2} \vee e_{1}\right)\right) \\
& +u_{1} u_{2}\left(\left(e_{1} \vee e_{3}\right)+\left(e_{0} \vee e_{2}\right)\right)
\end{aligned}
$$

We obtain the expression of $\mu^{\mathrm{s}}(u)$.
Let us prove Proposition 6 for oblique cameras. Consider an oblique camera whose linear congruence is denoted by $\mathcal{L}$ and whose retina $\mathcal{R}$ is endowed with a normalized basis $\left(\boldsymbol{y}_{0}, \boldsymbol{y}_{1}, \boldsymbol{y}_{2}\right)$. Let us define the points $\boldsymbol{p}_{i}$ as follows. We let $p_{0}=y_{0}$ and $p_{1}=y_{1}$. Since the ambiguity locus of the oblique camera is empty, there is a unique line $\ell^{\prime}$ of $\mathcal{L}$ through $\boldsymbol{y}_{2}$ which intersect one of the complex focal line defining $\mathcal{L}$ in a point of the form $\left[p_{2}+i p_{3}\right]$ where $p_{2}=$ $y_{2}$ and $p_{3}$ is real. According to Figure 11, we obtain the following basis of $\mathcal{L}_{\mathbb{C}}$ :

$$
\begin{aligned}
\xi_{0} & =p_{0} \vee p_{1} \\
\xi_{1} & =p_{2} \vee p_{3}, \\
\xi_{2}^{\prime} & =\left(p_{0}+i p_{1}\right) \vee\left(p_{2}-i p_{3}\right) \\
& =\left(p_{0} \vee p_{2}\right)+\left(p_{1} \vee p_{3}\right)-i\left(\left(p_{2} \vee p_{1}\right)+\left(p_{0} \vee p_{3}\right)\right) \\
\xi_{3}^{\prime} & =\left(p_{0}-i p_{1}\right) \vee\left(p_{2}+i p_{3}\right) \\
& =\left(p_{0} \vee p_{2}\right)+\left(p_{1} \vee p_{3}\right)+i\left(\left(p_{2} \vee p_{1}\right)+\left(p_{0} \vee p_{3}\right)\right)
\end{aligned}
$$

Considering $\xi_{2}=\left(\xi_{3}^{\prime}-\xi_{2}^{\prime}\right) / 2$ and $\xi_{3}=\left(\xi_{3}^{\prime}+\xi_{2}^{\prime}\right) / 2$, the family $\left(\xi_{i}\right)$ is a (real) basis of $\mathcal{L}$. Letting $M=\left[p_{0}, \ldots, p_{3}\right]$ and $\tilde{\boldsymbol{x}}=M \boldsymbol{x}$, we get that $\mathbf{M}$ maps $\mathcal{L}$ to the linear congruence of the standard camera (the basis $\left(\xi_{i}\right)$ is mapped to $\left(\xi_{i}^{\mathrm{s}}\right)$ ) and maps $\mathcal{R}$ to $\mathcal{R}^{\mathrm{s}}$ (the basis $\left(y_{i}\right)$ is mapped to $\left(y_{i}^{\mathrm{s}}\right)$ ). Up to projective transformation, the projection formulas are the standard one:

$\boldsymbol{u}=\left[\begin{array}{c}\tilde{x}_{0} \tilde{x}_{2}+\tilde{x}_{1} \tilde{x}_{3} \\ \tilde{x}_{1} \tilde{x}_{2}-\tilde{x}_{0} \tilde{x}_{3} \\ \tilde{x}_{2}^{2}+\tilde{x}_{3}^{2}\end{array}\right] \quad$ and $\quad \boldsymbol{\xi}=\left[\xi_{1}, \ldots, \xi_{4}\right]\left[\begin{array}{c}u_{0}^{2}+u_{1}^{2} \\ u_{2}^{2} \\ u_{0} u_{2} \\ u_{1} u_{2}\end{array}\right]$

and Proposition 6 for oblique cameras follows. 